ARTICLE

https://doi.org/10.1038/s41467-019-11120-0

\title{
Plasmodium myosin A drives parasite invasion by an atypical force generating mechanism
}

Julien Robert-Paganin', James P. Robblee², Daniel Auguin (10 ${ }^{3}$, Thomas C. A. Blake4, Carol S. Bookwalter², Elena B. Krementsova², Dihia Moussaoui ${ }^{1}$, Michael J. Previs², Guillaume Jousset', Jake Baum (1) ${ }^{4}$, Kathleen M. Trybus ${ }^{2} \&$ Anne Houdusse (iD ${ }^{1}$

Plasmodium parasites are obligate intracellular protozoa and causative agents of malaria, responsible for half a million deaths each year. The lifecycle progression of the parasite is reliant on cell motility, a process driven by myosin $A$, an unconventional single-headed class XIV molecular motor. Here we demonstrate that myosin A from Plasmodium falciparum (PfMyoA) is critical for red blood cell invasion. Further, using a combination of $X$-ray crystallography, kinetics, and in vitro motility assays, we elucidate the non-canonical interactions that drive this motor's function. We show that PfMyoA motor properties are tuned by heavy chain phosphorylation (Ser19), with unphosphorylated PfMyoA exhibiting enhanced ensemble force generation at the expense of speed. Regulated phosphorylation may therefore optimize PfMyoA for enhanced force generation during parasite invasion or for fast motility during dissemination. The three PfMyoA crystallographic structures presented here provide a blueprint for discovery of specific inhibitors designed to prevent parasite infection.

\footnotetext{
${ }^{1}$ Structural Motility, UMR 144 CNRS/Curie Institute, 26 rue d'ulm, 75258 Paris cedex 05, France. ${ }^{2}$ Department of Molecular Physiology and Biophysics, University of Vermont, Burlington, VT 05405, USA. ${ }^{3}$ Laboratoire de Biologie des Ligneux et des Grandes Cultures (LBLGC), Université d'Orléans, INRA, USC1328, 45067 Orléans, France. ${ }^{4}$ Department of Life Sciences, Imperial College London, Exhibition Road, South Kensington, London SW7 2AZ, UK. Correspondence and requests for materials should be addressed to K.M.T. (email: kathleen.trybus@uvm.edu) or to A.H. (email: anne.houdusse@curie.fr)
} 
$\mathrm{M}$ alaria is a mosquito-borne disease caused by obligate protozoan parasites from the genus Plasmodium. Despite global efforts to control the disease, malaria is still responsible for half a million deaths each year, with the vast majority of mortality caused by $P$. falciparum ${ }^{1}$. During its complex life-cycle, the parasite transforms through motile and non-motile stages in its two hosts: human and mosquito (Supplementary Fig. 1a). The sporozoite stage that establishes infection, is injected into the dermis following the bite of an infected female mosquito from the genus Anopheles. This elongated and highly motile parasite form reaches dermal capillaries and is rapidly transported to the liver, where it targets and infects hepatocytes. Within hepatocytes the parasite grows and divides, eventually releasing tens of thousands of merozoite forms en masse into the blood stream. Merozoites, in contrast to sporozoites, are relatively non-motile stages that specifically target erythrocytes, where they develop and lead to all symptoms associated with malaria disease. Beyond this asexual replicative stage of development, some parasites switch commitment to develop into sexual forms (following an as yet unknown signal) producing male and female gametocytes that re-establish mosquito infection on the next bite $^{2}$ (Supplementary Fig. 1a). Whilst anti-malarials are available, resistance to all frontline drugs, including combination therapies based on artemisinin, continues to emerge ${ }^{3}$ (Supplementary Fig. 1a), reinforcing the need to find drugs with new modes of action.

Force production and movement are essential for the progression at each stage of the parasite's two host life-cycle. Erythrocytic invasion by a merozoite, for example, involves a force of $\sim 40 \mathrm{pN}^{4}$, while sporozoites can move at a speed of $2 \mu \mathrm{m} . \mathrm{s}^{-1}$, an order of magnitude faster than the fastest human immune cells ${ }^{5}$. Like all Apicomplexa (the phylum to which Plasmodium belong), malaria parasites move using a substrate-dependent mechanism called gliding motility, a sophisticated substrate-dependent form of cell movement based on a macromolecular complex called the glideosome (Supplementary Fig. 1b). At the glideosome core is the single-headed class XIV myosin A (PfMyoA), and short and oriented filaments of a divergent actin (PfAct1). PfMyoA is a tailless myosin consisting only of a motor domain and light chain binding domain. The $\mathrm{N}$-terminal region of one of the light chains (LC), called myosin tail interacting protein (MTIP), is believed to anchor the myosin to a membrane bound complex of glideosome associated proteins (GAP45-GAP50-GAP40) (Supplementary Fig. 1b). Several studies have investigated the role of myosin A in both the coccidian parasite Toxoplasma gondii and across the genus Plasmodium. In Toxoplasma gondii, Myosin A (TgMyoA) has been implicated in both invasion and egress of the parasite from the infected cell ${ }^{6,7}$. TgMyoA is not, however, essential for invasion and its function can be compensated for by TgMyoB or its splicing isoform $\mathrm{TgMyoC}^{8,9}$, or by active host cell-mediated internalization ${ }^{10}$. Although host cell membrane wrapping forces likely play a role in merozoite invasion of red blood cells ${ }^{11}$, their role has not been tested in the absence of a parasite motor. In the Plasmodium genus, initial experiments using the mouse malaria parasite $P$. berghei, showed that $\mathrm{PbMyoA}$ is required for cell motility and midgut colonization during parasite mosquito stages $^{12}$. More recently, genetic ablation of GAP45 in P. falciparum blocked parasite invasion in merozoite stages, but not egress ${ }^{13}$. Whilst this study points to a critical role for the motor complex in blood stages of infection, the structural role GAP45 plays in apicomplexan cell architecture and glideosome function leaves the essential functionality of MyoA in the merozoite unresolved.

Given that the core of the glideosome is composed of divergent forms of both myosin and actin, the actomyosin system of $P$. falciparum is an attractive target for generation of new anti-malarial drugs. Actin is a highly conserved, ubiquitous protein in all Eukaryotes, with yeast and human actins sharing $87 \%$ identity, in contrast to $<80 \%$ identity of PfAct1 with canonical actins. The sequence of PfAct1 differs from canonical actins in regions implicated in inter-protomer interfaces of the actin filament ${ }^{14}$. Accordingly, high resolution cryo-electron microscopy (CryoEM) studies confirmed altered inter-strand and intrastrand contacts that provided a structural basis for the instability of PfAct1 filaments ${ }^{14,15}$. PfMyoA is also a divergent motor, sharing only $30 \%$ sequence identity with Class II myosins. Interestingly, important sequence differences are located precisely in structural elements predicted to be relaying parts of the allosteric communication pathway within the myosin motor domain. In Class XIV myosins, to which PfMyoA belongs, the sequence of critical canonical residues for the motor mechanism are not conserved, including a pivotal ${ }^{\mathrm{SH} 2}$-SH1Gly (G695 in scallop myosin 2) at the beginning of the SH1-helix. The connectors (Switch-2, the Relay helix, and the SH1-helix) are structural elements that coordinate rearrangements of the four motor subdomains, whose motions are amplified into a larger swing of the distal light chain binding lever arm (Supplementary Fig. 2). Mutations of this ${ }^{\mathrm{SH} 2}-\mathrm{SH}_{1} \mathrm{Gly}$ greatly impede motor activity in class 2 myosins (Myo2) by reducing the flexibility of the fulcrum ${ }^{16-18}$. Despite lacking this fulcrum, PfMyoA nonetheless displays robust motor activity ${ }^{19}$. Resolving this conundrum requires structural studies of MyoA in different nucleotide states to reveal the key compensatory intra-molecular rearrangements that are involved in guiding the lever arm. Such a force generation mechanism is likely to be unique to the apicomplexan class XIV myosins. Based on a recently solved crystal structure of only one state (Pre-powerstroke, PPS) of the TgMyoA motor domain with a disordered $\mathrm{N}$-terminal region, Boulanger and coworkers could only speculate about how sequence adaptations in the Wedge, the Relay and the fulcrum (Supplementary Fig. 2) may compensate for this mechanism ${ }^{20}$.

By combining parasitological studies of MyoA in P. falciparum with structural, motility, and kinetic studies of the motor in vitro, we show that PfMyoA is critical for $P$. falciparum erythrocyte invasion, and that this atypical motor diverges significantly with regard to how force and motion is produced compared with conventional myosins. By solving three structural states of the PfMyoA motor domain, corresponding to three states of the motor cycle, we show how the unique class XIV N-terminal heavy chain extension and its interactions with other structural elements define motor function. We also show that Ser19 phosphorylation in this $\mathrm{N}$-terminal extension substantially modulates the speed and force output generated by the motor. These insights allow us to propose a phosphorylation-dependent mechanism that tunes the motor for optimal invasion (high force) or dissemination (fast motility speed). Together, these insights provide a complete foundation from which to understand the noncanonical mechanism of force production in these globally significant parasites.

\section{Results}

PfMyoA is critical for erythrocytic invasion by merozoites. Whilst GAP45 has been reported to be essential for erythrocytic invasion by merozoites ${ }^{13}$ the role of the gliding motor itself, PfMyoA, in Plasmodium blood stages, has never been directly tested. To address this question, we generated a rapamycin (RAP) inducible PfMyoA KO ${ }^{21}$ (Fig. 1a). Rapamycin treatment induces a constitutively expressed DiCre recombinase, leading to excision of the sequence between two loxP sites integrated into the pfmyoa gene $^{22}$, deleting the last 543 bases and bringing $g f p$ into frame as a truncated fusion protein (residues 1-638). The resulting truncated PfMyoA motor lacks the C-terminal residues predicted 
a

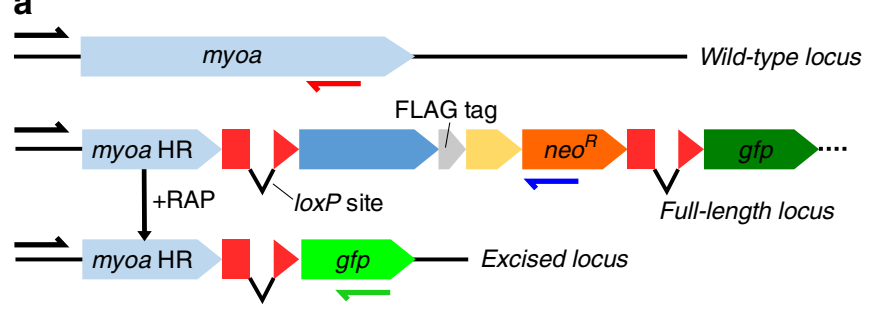

C
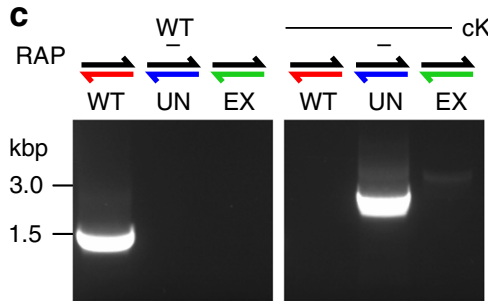

cKO

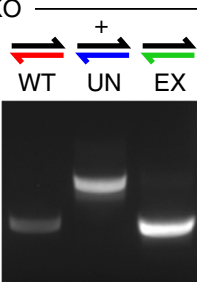

d
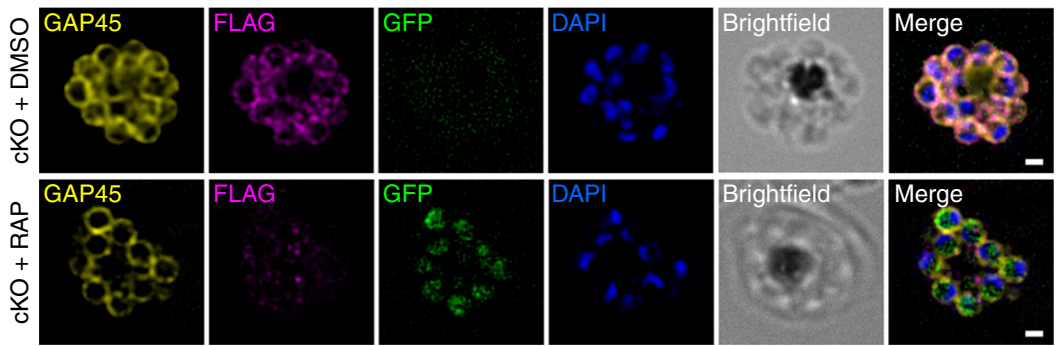

b

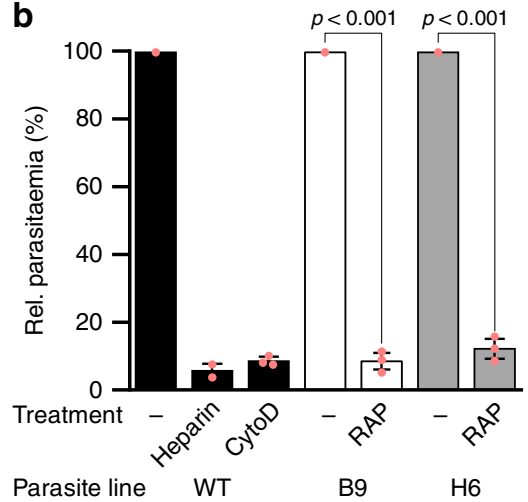

e
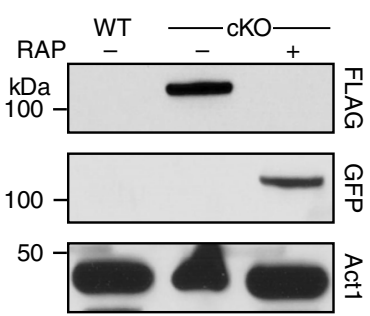

Fig. 1 PfMyoA is critical for red blood cell invasion by merozoites. a Schematic showing replacement of the wild-type myoa locus with the full-length mutant locus by single crossover recombination in the myoa HR region using a T2A skip peptide (yellow box) to couple genomic integration to neomycin selection. b Treatment of two MyoA-cKO clones (B9 and $\mathrm{H} 6$ ) or WT with rapamycin (RAP) shows an almost complete invasion block ( $90 \%)$ compared to DMSO treatment (-). This is comparable to the defect with known invasion inhibitors heparin and Cytochalasin D (CytoD) (90-95\%). Parasitaemia was measured in the following cycle (cycle 1) by flow cytometry as the percentage of red blood cells (RBCs) that were DNA-positive by staining with SYBR Green I. Mean of three biological replicates (except for WT+heparin: two biological replicates), each three technical replicates, \pm standard deviation (S.D.) of biological replicates. Data from each biological replicate were normalized to the DMSO-treated sample for each parasite line. Significance assessed using parametric t-test (paired, two-tailed). c Genotyping PCR of WT or cKO parasites following DMSO (-) or RAP (+) treatment detecting the wild-type (WT, red half-arrow), unexcised (UN, blue half-arrow) and excised (EX, green half-arrow) pfmyoa loci. d Immunofluorescence analysis of cKO parasites following DMSO or RAP treatment. FLAG-tag is detectable in DMSO-treated schizonts fixed $\sim 48 \mathrm{~h}$ post-treatment, colocalising with motor complex protein GAP45, while after RAP treatment GFP but not FLAG is detectable, and the signal is restricted to the cytosol, consistent with a non-functional truncated MyoA. Scale bar $1 \mu \mathrm{m}$. Image stacks were deconvolved using the EpiDEMIC plugin for Icy, with a z-step size of $200 \mathrm{~nm}$. e Western blot of WT and cKO parasites following DMSO (-) or RAP (+) treatment. Parasites were lysed $40 \mathrm{~h}$ post-treatment, before the end of cycle 0 . In DMSO-treated cKO parasites, the original FLAG-tag is detectable, but following RAP-treatment, only GFP is detectable. PfAct1 was used as a loading control. Representative blot shown from three biological replicates

to be required for the folding of the motor domain and would therefore be expected to be non-functional.

Two PfMyoA-cKO clones (B9 and H6) were compared with both WT and non-excised (DMSO treatment) controls to assess motor function. In addition, two known invasion inhibitors, heparin (that blocks cell-to-cell interactions) and CytoD (that blocks actin polymerization) served as positive controls ${ }^{23}$ (Fig. 1b). Synchronized parasites were treated with $100 \mathrm{nM}$ RAP and incubated for $16 \mathrm{~h}$ at the start of one cycle (cycle 0 ) to trigger truncation of pfmyoa. These were then tested for invasion of red blood cells (RBCs). Parasitaemia was measured in the following cycle (cycle 1) by flow cytometry as the percentage of RBCs that were DNA-positive by staining with SYBR Green I (Fig. 1b). Successful excision of the loxP section of pfmyoa was monitored by PCR (Fig. 1c), localization of PfMyoA by immunofluorescence (Fig. 1d) and western blotting (Fig. 1e), all confirming loss of the functional protein.

The KO of PfMyoA induced in the two PfMyoA-cKO clones demonstrated an almost complete block in invasion ( $90 \%)$ compared to DMSO treatment, comparable to rates of invasion detected with known invasion inhibitors heparin and CytoD (90-95\%) (Fig. 1b). This confirms the long-held assumption that PfMyoA is directly involved in merozoite invasion of the erythrocyte in P. falciparum. Given the combined evidence that MyoA is critical for both invasion and for motility ${ }^{12}$ in Plasmodium parasites, it would appear to be a bona fide firstorder therapeutic target for preventing malaria parasite infection and disease progression.

X-ray structures of three structural states of PfMyoA. To define the motor cycle of PfMyoA, we determined the crystallographic structures of the motor domain (MD) of PfMyoA (1-768) in a nucleotide-free (NF) condition at $2.82 \AA$ resolution (crystal type 1) (Supplementary Table 1, PDB code 6I7D) and complexed to ADP.Vanadate at $3.45 \AA$ resolution (crystal type 2) (Supplementary Table 2, PDB code 6I7E). Four molecules are present in the asymmetric unit of crystal type 1 , one of the motor domain is 
in the Rigor-like state and three molecules adopt the Post-rigor (PR) state. These two structural states correspond, respectively to the state of highest affinity for F-actin ${ }^{24,25}$ (Rigor-like) and to the state that allows detachment from F-actin but prior to the lever arm recovery stroke ${ }^{26}$ (Post-rigor). The PfMyoA Rigor-like state adopts a fully closed cleft as found for non muscle Myo2c and Myolb Rigor states ${ }^{25,27}$ and is thus likely a good model of myosin XIV in the Rigor state.

The crystal type 2 contains one molecule per asymmetric unit. It corresponds to the Pre-powerstroke (PPS) state, a state with the lever arm up and able to dock to actin with electrostatic contacts. The resolution of the PPS state is poorer, but the electron density map is of good quality and allowed the entire motor domain to be built, including loops with good refinement statistics (Supplementary Table 2). Description of these three structural states for the PfMyoA motor provides a direct way to assess the major conformational changes that would occur during the motor cycle (Fig. 2a).

These structures enable visualization of the large conformational changes required for the powerstroke (PPS/Rigor), for detachment of the motor from actin (Rigor/PR), and for repriming of the lever arm (PR/PPS). The amplitudes of the subdomain movements and converter swing are similar to that of other myosins ${ }^{28}$ (Fig. 2a), but the stabilization of the states and the connector rearrangements differ. A key observation we made is the role of the unusual $\mathrm{N}$-terminal (N-term) extension of PfMyoA (Fig. 2b, Supplementary Movie 1). This extension makes conserved interactions with the $\mathrm{N}$-term subdomain in all three structures, and consists of a helix followed by a linker that contains the phosphorylated Ser19 (SEP19) (Supplementary Fig. 3). Structural analysis presented in detail below shows an unforeseen compensatory mechanism that involves the $\mathrm{N}$-term extension driving the PfMyoA powerstroke despite the lack of sequence conservation in canonical residues of the motor domain connectors (Fig. 3a).

Motor mechanism in classical myosins. During motor domain rearrangements required for the motor cycle, movement near the active site involves the connector Switch-2, and is transmitted to the lever arm through interactions between Switch-2 and an important structural element of the Lower $50 \mathrm{kDa}$ (L50) subdomain called the Wedge (Fig. 3b, Supplementary Fig. 2, 4). To drive the lever arm swing in all myosins studied thus far (classical myosins), these Switch-2 rearrangements are coordinated with specific changes of two other connectors: the Relay helix kink straightening and a SH1-helix rotation ${ }^{28}$ (Fig. 3c, Supplementary Fig. 4, Supplementary Movie 2). The highly conserved glycine

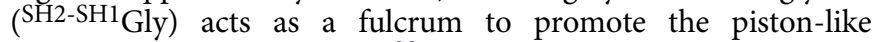
movement of the SH1-helix ${ }^{29}$. Conserved interactions between the Relay/SH1-helix and the Wedge/Switch-2 are critical for motor activity (Fig. 3, Supplementary Fig. 5, Supplementary Movies 2 and 3). In classical myosins, such as scallop myosin II (ScMyo2) (Supplementary Fig. 5a), the active site rearrangements

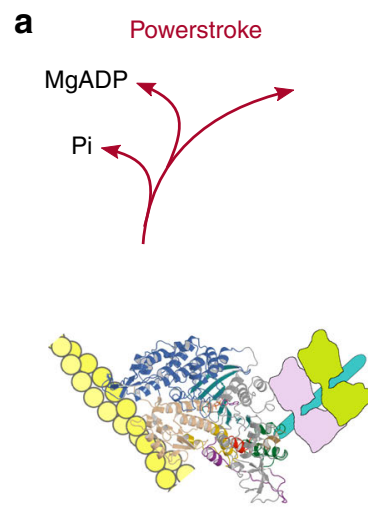

Actin-bound Pre-Powerstroke

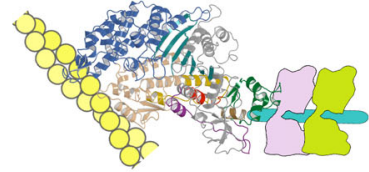

Rigor-like
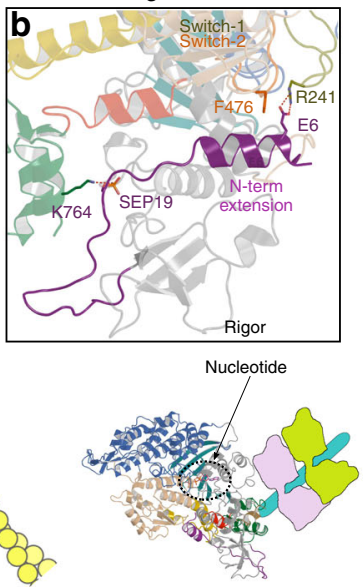

Pre-Powerstroke
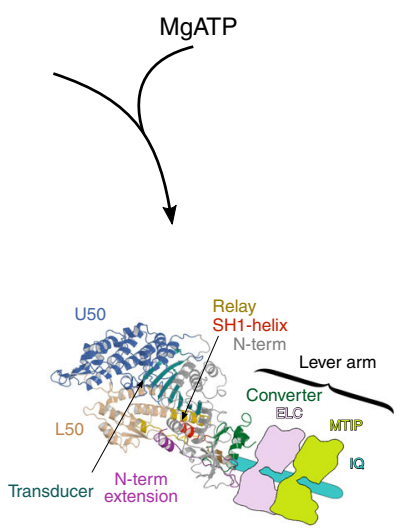

Post-Rigor

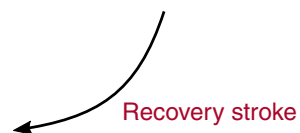

Fig. 2 Structural states and motor cycle of PfMyoA. a The crystallographic structures of the three states of the motor cycle of PfMyoA are represented along the motor cycle: the Rigor-like and the Post-rigor (PR) states reveal how the motor detaches from F-actin upon ATP binding; the Pre-powerstroke (PPS) state corresponds to the state in which hydrolysis occurs and that rebinds to F-actin to trigger the powerstroke. The Rigor-like state is the conformation the motor adopts at the end of the powerstroke when hydrolysis products have been released. To appreciate the movement of the converter and how it can be amplified by the rest of the lever arm, the IQ region and the two LCs (PfELC and MTIP, Supplementary Fig. 1) are represented schematically in continuity of the last helix of the converter. PfMyoA displays the four canonical subdomains which are the hallmark of the myosin superfamily: N-terminus (N-term) (gray), Upper 50 kDa (U50) (marine blue), Lower 50 kDa (L50) (tint) and the converter (green) and central elements (including a beta-sheet) forming the transducer (dark cyan). During the motor cycle, rearrangements in the motor domain are allosterically transmitted through the Relay helix (yellow) and are amplified by the swing of the converter with the rest of the lever arm. $\mathbf{b}$ In the center diagram, a zoom of the Rigorlike structural state is presented to show the opposite side of the motor domain compared to a. Note the position of the unique N-terminal extension (purple) which is close to the connectors that direct rearrangements between motor subdomains (Switch-2 (orange), Relay (yellow) and SH1-helix (red)). PfMyoA employs an atypical motor mechanism in which the $\mathrm{N}$-term extension (purple) compensates for non-canonical sequences in subdomain connectors essential for motor function 


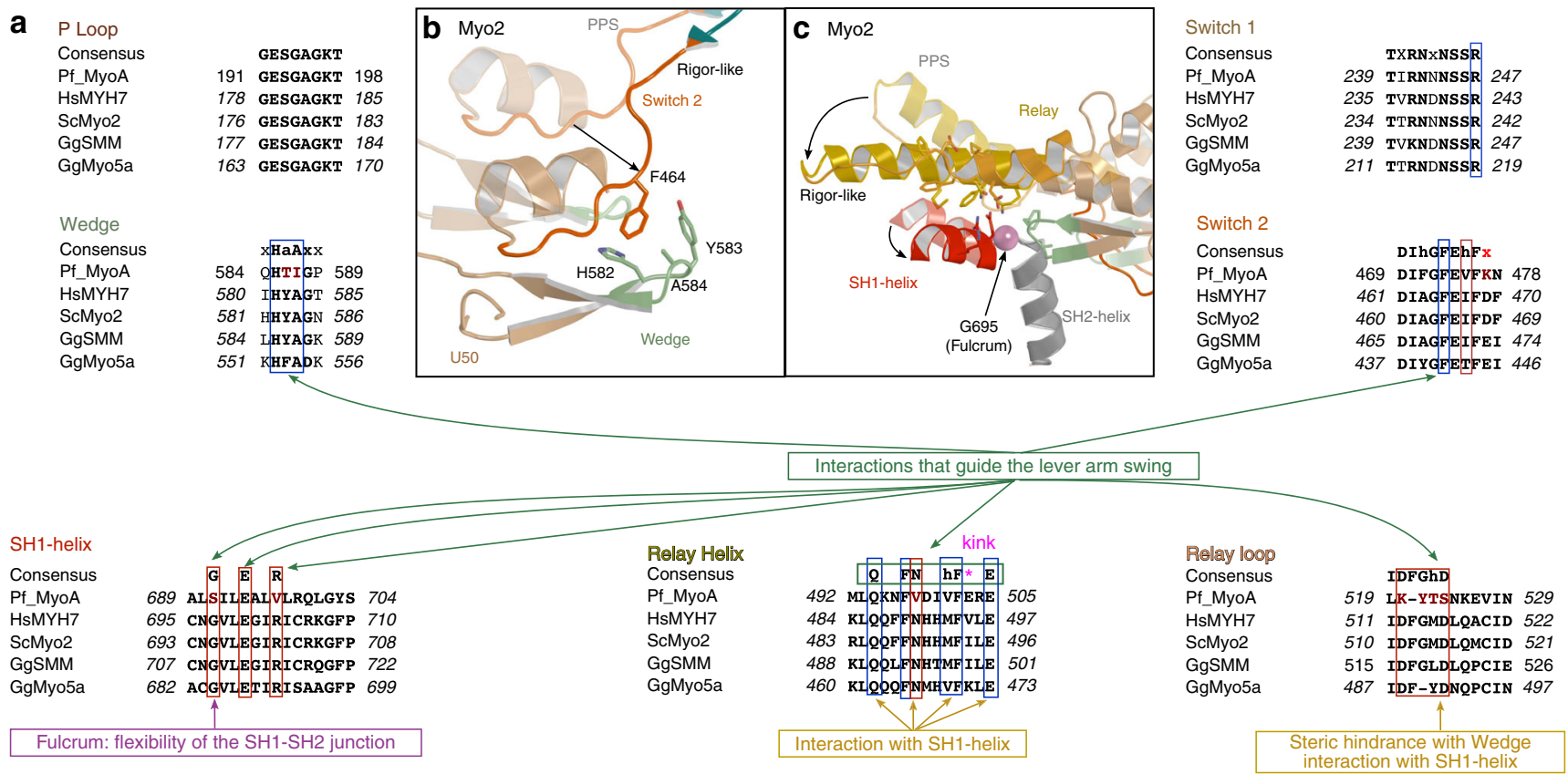

Fig. 3 Sequence alignment of connectors essential in driving motor conformational changes-lack of canonical residues in PfMyoA. a Sequence comparison of several key elements involved in myosin mechanical transduction and allosteric communication. The consensus is represented on top of each sequence and the PfMyoA sequence is compared to other myosins. This comparison shows that the sequences of the Switch-2, Wedge, and Relay differ from the consensus. Consensus code: $\mathrm{a}$ is an aromatic residue, $\mathrm{h}$ a hydrophobic residue, $\mathrm{x}$ colored in red is an acidic residue. Residues important in allosteric communication in the motor are indicated in rectangles (blue rectangle if the residue is involved in a conserved interface, red rectangle if the residues are involved in interactions specific to PfMyoA). If the position is not conserved in PfMyoA, the residue is colored in dark red. To see how these residues affect the PfMyoA motor rearrangements along the cycle, see Fig. 4 and Supplementary Movie 2. b Switch-2/Wedge interactions in classical Myo2. c Conformational changes of two connectors before (PPS, transparent) and after (Rigor-like, plain) the powerstroke in Myo2. The Relay helix unkinks while

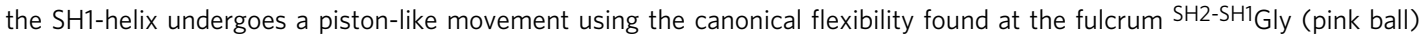

are linked to Switch-2 changes that are guided by hydrophobic interactions of Switch-2 F464 with canonical residues of the Wedge such as Wedge H582 and Wedge A584 (Supplementary Fig. 5b). In the PPS state, the Relay, the SH1-helix and the lever arm are in a "primed" conformation: the interactions between the Relay and the SH1-helix are mainly hydrophobic. Here the conserved RelayM514 and SH1-helixE698 are nearby (Supplementary Fig. 5b). During the powerstroke, release of hydrolysis products induces specific structural rearrangements in the active site, and specifically of Switch-2 that repositions the L50 subdomain. In so doing, the Wedge which belongs to the L50 subdomain becomes closer to the Relay/SH1-helix position. The steric hindrance caused by a conserved bulky aromatic, WedgeY583 with residues from the SH1-helix and the Relay, RelayM514 and SH1-helixE698, drives the straightening of the Relay, the piston-like movement of the SH1helix (Supplementary Fig. 5b, Supplementary Movies 2 \& 3) and thus the lever arm swings because these two connectors interact strongly with the converter (Supplementary Fig. 5c). The movement of the SH1-helix and the Relay are defined by interactions between these two connectors which are not only hydrophobic but supplemented with electrostatic interactions between conserved residues in the Rigor-like state: the conserved SH1-helix R701 binds RelayD515; SH1-helixE698 binds RelayQ485; and RelayN489 (Supplementary Fig. 5b).

The atypical structural motor mechanism of PfMyoA. In PfMyoA, the amplitude of the converter swing is similar to that observed for classical myosins, despite the absence of the canonical residues essential for allosteric movement in classical myosins. This occurs while the SH1-helix is mostly immobile (Fig. 4), rather than performing a piston-like movement previously described for classical myosins (Supplementary Fig. 5, Supplementary Movies 2 and 3). As predicted from sequence alignments, the replacement of the conserved ${ }^{\mathrm{SH}} 2-\mathrm{SH} 1$ Glycine restrains the mobility of the $\mathrm{SH} 1$ helix. Several sequence adaptations are needed to compensate for the lack of mobility of the SH1-helix. First, the aromatic and bulky residue of the Wedge is absent in PfMyoA and replaced by a threonine (T586), and the nearby bulky methionine residue from the Relay (Relay511-DFGMD-515 in ScMyo2) is also absent and replaced by a threonine (T522) in PfMyoA (Relay520-KYTS-523) (compare Fig. 4b and Supplementary Fig. 5b; Supplementary Movie 4). The movement of the Wedge during the powerstroke results in less steric hindrance and is thus adapted to the lack of mobility of the SH1-helix which stays in position while the Relay kink resolves and the lever arm swings (Fig. 4b, c, Supplementary Movie 3). Second, the interaction between the Relay and the SH1-helix is mainly hydrophobic both in the PPS state and in the Rigor-like state, with the exception of an electrostatic bond between the RelayQ494 and the fulcrum ${ }^{\mathrm{SH} 2-\mathrm{SH}}{ }^{\mathrm{S}} 691$ that is present in the two states (Fig. 4b, Supplementary Movie 3). Third, the PfMyoA Rigor-like state is stabilized by atypical interactions of WedgeI587

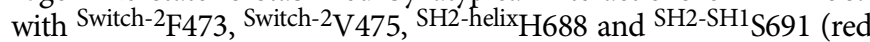
circle in Fig. 4b). Additional interactions with the N-term extension further stabilize the Rigor-like state, including a strong salt bridge- $\pi$ interaction between the N-term.extension Glu6, Switch-1 Arg241 and Switch-2 Phe476 (Fig. 4c). In addition, electrostatic contacts directly stabilize the converter since ConverterK764 interacts with the phosphorylated N-term.extensionSEP19 (Fig. 4c).

We conclude from the structures that the electrostatic interactions with the $\mathrm{N}$-term extension compensate for the absence of a piston-like movement of the SH1-helix, enabling force production by accelerating the transitions of the 


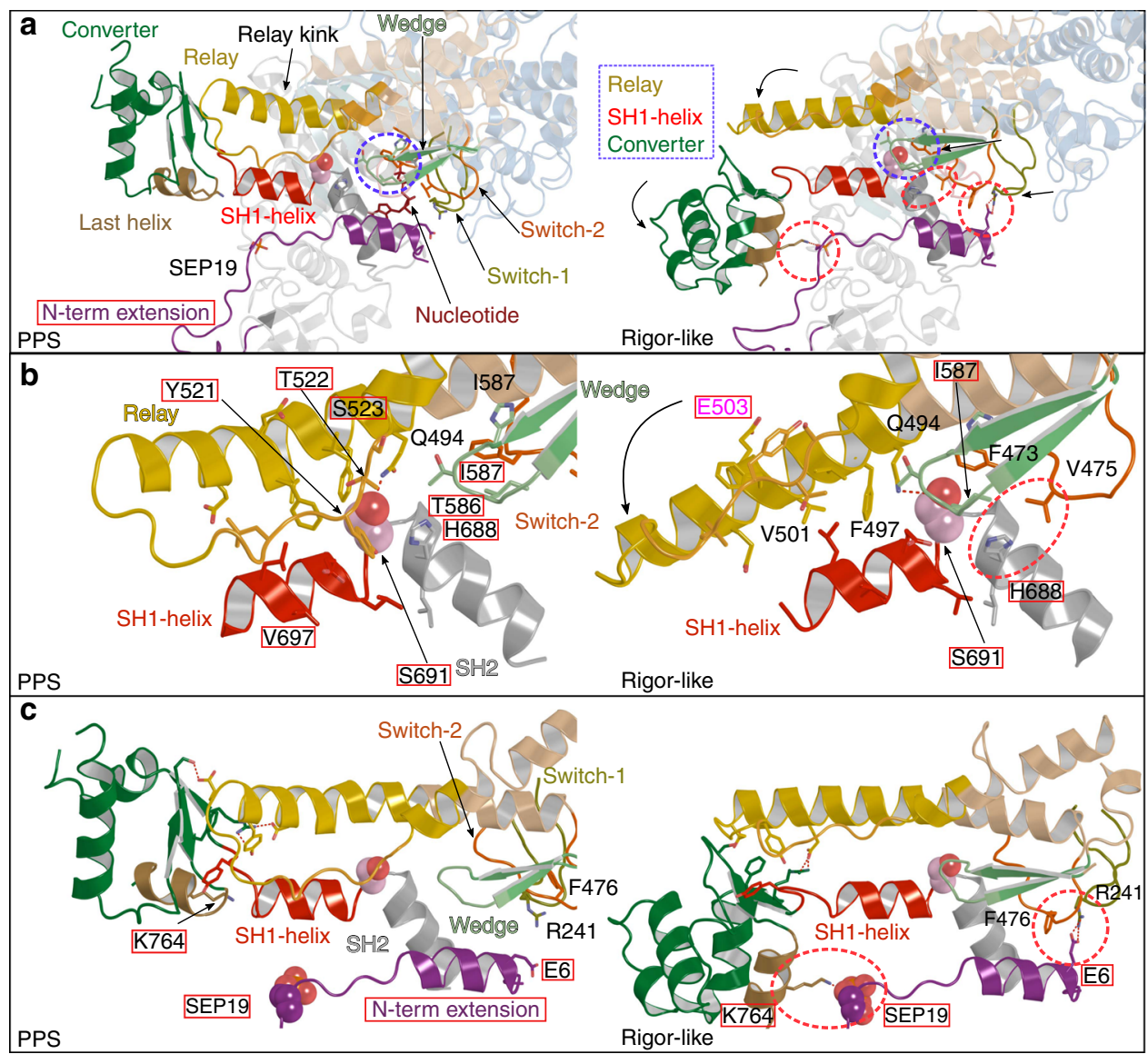

Fig. 4 The unconventional mechanism of force production by PfMyoA. a Overall view of the mechanical communication within the PfMyoA motor domain during the powerstroke. In both the PPS and the Rigor-like states, interactions between Switch-2 and the Wedge are maintained (highlighted by dashed blue lines and detailed in $\mathbf{b}$ ). The Rigor-like structure indicates that the sequential release of hydrolysis products upon the powerstroke triggers displacement of the Wedge which is associated with straightening of the Relay helix and the converter swing. The PfMyoA unconventional powerstroke

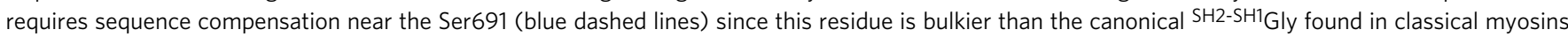
at this position. Thus, motor domain rearrangements are allowed by changes in the interactions between the Wedge and the Relay and SH1-helix connectors (details in $\mathbf{b}$ ). Additional interactions (highlighted by dashed red lines) involving the $\mathrm{N}$-term extension (purple) also stabilize the Rigor-like state and compensate for the immobility of the SH1-helix. (Details are shown using the same view in $\mathbf{c}$ ). $\mathbf{b}$ Non-conserved residues are highlighted by a red

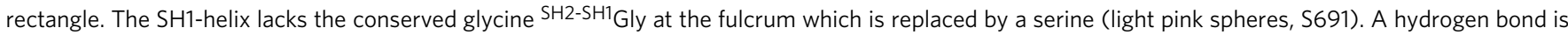
formed between $\mathrm{S} 691$ and Relay 494 in both the PPS and Rigor-like states. The presence of a less pliant fulcrum requires sequence adaptation in the Wedge and in the Relay and results in the immobility of the SH1-helix during the powerstroke. Switch-2 V475 establishes hydrophobic interactions with $\mathrm{SH}_{2} \mathrm{H} 688$, helping to stabilize the Rigor-like position of the Switch-2 (red dashed lines). E503 (shown in pink) is a reporter to indicate the kink of the Relay. c In PfMyoA, the converter establishes a network of interactions with the Relay and the SH1-helix. Non-conserved residues are highlighted by a red rectangle. An electrostatic bond between phosphoserine 19 (SEP19) ( $N$-term extension) and K764 (converter), as well as a salt bridge- $\pi$ interaction between the N-term extensionE6, Switch-1R241, Switch-2F476 (red dashed lines) stabilize the position of the converter in the Rigor-like conformation. For comparison with Myo2, see Supplementary Fig. 5 and Supplementary Movies 2 and 3

powerstroke. In particular, these interactions could accelerate ADP release which was slowed when the ${ }^{\mathrm{SH} 2-\mathrm{SH} 1}$ Gly was mutated in a canonical Myo2 motor ${ }^{17,18}$.

PfMyoA phosphorylation tunes its motor properties. To further analyze the compensatory mechanism and more fully decipher the role of the N-term phosphorylation, we functionally characterized PfMyoA constructs with a 19 amino acid N-term deletion $(\Delta \mathrm{N})$, or with point mutations that disrupt the N-term extension/converter interaction formed in the Rigor-like state (phospho-null S19A or K764E) (Figs. 4c, 5a). In agreement with the phosphorylated Ser (SEP19) observed in the crystallographic structures described above, we verified that PfMyoA was phosphorylated at Ser19 on the heavy chain during expression in Sf9 cells. Phosphoprotein gels qualitatively showed heavy chain phosphorylation, and mass spectrometry confirmed that $96 \pm 1 \%$
( $\pm S D, n=6,2$ independent preparations) of the peptides found in the untreated sample were phosphorylated compared with a phosphatase-treated sample (Supplementary Fig. 6).

In vitro motility assays showed that the $\mathrm{N}$-term extension and, specifically the Ser19 phosphorylation, are necessary for actin displacement at maximal speed $\left(\sim 4 \mu \mathrm{m} . \mathrm{s}^{-1}\right) . \Delta \mathrm{N}$-PfMyoA reduced speed $\sim 17$-fold, while both the phospho-null (S19A) and charge reversal mutation in the converter (K764E) mutants slowed speed 2-fold to $\sim 2 \mu \mathrm{m} . \mathrm{s}^{-1}$ (Fig. 5b, Supplementary Table 3). The phosphomimic S19E did not fully recapitulate the enhancing effect of bona fide phosphorylation on speed, moving actin at a value intermediate $(\sim 2.8 \mu \mathrm{m} / \mathrm{s})$ between $\mathrm{S} 19 \mathrm{~A}$ and phosphorylated PfMyoA (Supplementary Fig. 7a).

The same pattern seen for in vitro motility was also observed for the maximal rate of the steady-state actin-activated ATPase activity (WT $>$ S19A or K764E $>\Delta N$ ) (Fig. 5c). A linked assay with 
a

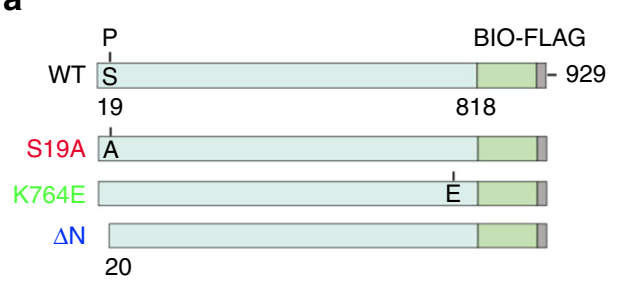

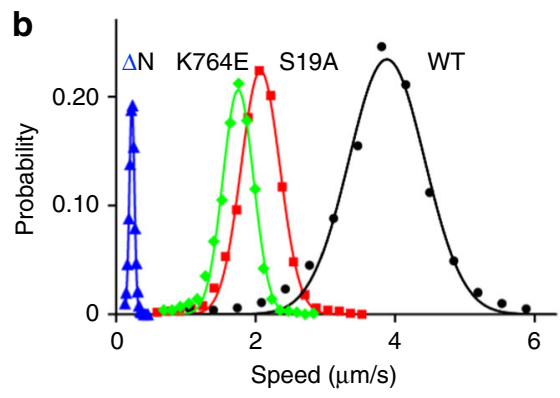

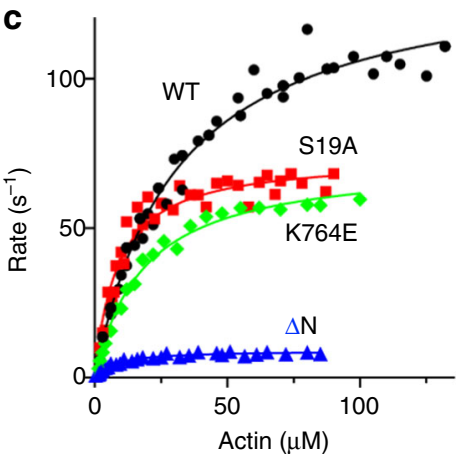

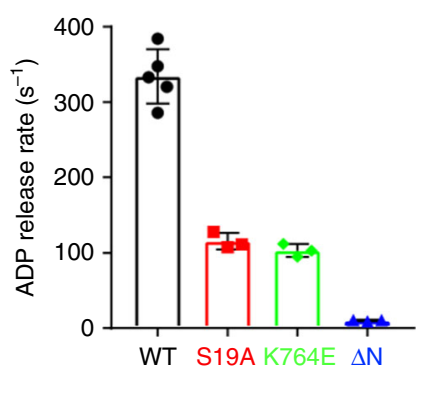

e

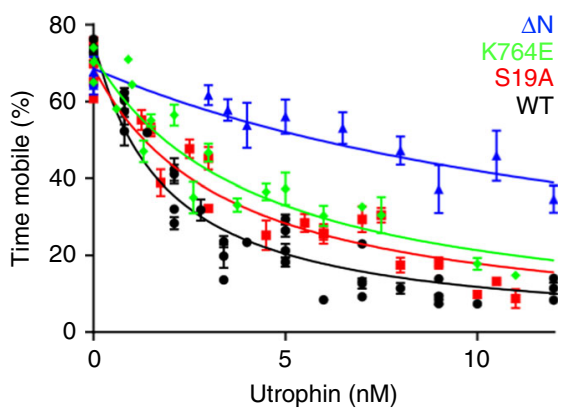

Fig. 5 Functional properties of wild-type (WT) and mutant full-length PfMyoA constructs. a Schematic of constructs used for functional analysis. Ser19 is fully phosphorylated during protein expression in Sf9 cells (Supplementary Fig. 6). b Speed distributions from a representative in vitro motility assay. WT, $3.88 \pm 0.54 \mu \mathrm{m} / \mathrm{s} ; \mathrm{S} 19 \mathrm{~A}, 2.07 \pm 0.28 \mu \mathrm{m} / \mathrm{s} ; \mathrm{K} 764 \mathrm{E}, 1.75 \pm 0.22 \mu \mathrm{m} / \mathrm{s}$; and $\Delta \mathrm{N}, 0.23 \pm 0.04 \mu \mathrm{m} / \mathrm{s}$. Values, mean $\pm \mathrm{SD}$ (Supplementary Table 3 shows data from multiple protein preparations). c Actin-activated ATPase activity for WT, $V_{\max }=138 \pm 4 \mathrm{~s}^{-1}$ and $\mathrm{K}_{\mathrm{m}}=30.3 \pm 2.3 \mu \mathrm{M} ; \mathrm{S} 19 \mathrm{~A}, \mathrm{~V}_{\max }=74.0 \pm 2.0 \mathrm{~s}^{-1}$ and $K_{m}=8.5 \pm 1.0 \mu M ; K 764 E, V_{\max }=72.9 \pm 1.9 \mathrm{~s}^{-1}$ and $K_{m}=18.2 \pm 1.4 \mu \mathrm{M}$; and $\Delta \mathrm{N}, \mathrm{V}_{\max }=9.13 \pm 0.20 \mathrm{~s}^{-1}$ and $\mathrm{K}_{\mathrm{m}}=7.34 \pm 0.67 \mu \mathrm{M}$. Data from 2 protein preparations and 3 experiments for each construct were fitted to the Michaelis-Menten equation. Error, SE of the fit. d ADP release rates from actoPfMyoA. WT, $334 \pm 36 \mathrm{~s}^{-1}$; S19A, $115.8 \pm 10.9 \mathrm{~s}^{-1} ; \mathrm{K} 764 \mathrm{E}, 103.5 \pm 8.6 \mathrm{~s}^{-1} ; \Delta \mathrm{N}, 10.32 \pm 0.91 \mathrm{~s}^{-1}$. Values, mean $\pm \mathrm{SD}$. WT vs. any other construct. $\mathrm{p}<0.0001$; S19A vs. K764E, NS; S19A or K764E vs. $\Delta N, p<0.01$ (one way ANOVA followed by a Tukey's Honest Significant Difference post-hoc test). Data from at least 3 protein preparations of each construct at different temperatures are shown in Supplementary Table 4. e Ensemble force measurements using a utrophin-based loaded in vitro motility assay. A myosin that produces more force requires higher utrophin concentrations to arrest motion: WT, $1.40 \pm$ $0.08 \mathrm{nM}$; S19A, $2.42 \pm 0.17 \mathrm{nM} ; \mathrm{K} 764 \mathrm{E}, 3.04 \pm 0.30 \mathrm{nM} ; \Delta \mathrm{N}, 10.8 \pm 0.8 \mathrm{nM}$. Error, SE of the fit. Data from two protein preparations and three experiments for each construct. Supplementary Fig. $7 \mathrm{~b}$ shows these force data and fits extended to higher utrophin concentrations. Supplementary Fig. 7c-e shows $\Delta \mathrm{N}$ data shown with an expanded y-axis. Skeletal actin was used for all experiments. Temperature, $30^{\circ} \mathrm{C}$. Source data are provided as a Source Data file

an ATP regenerating system was used to prevent ADP buildup during the course of the assay, which could potentially slow the ATPase rate. Transient kinetics were used to directly measure the rate of ADP release from acto-PfMyoA. A high concentration of MgATP was mixed with acto-PfMyoA (MgADP). Under these conditions, the rate of dissociation of PfMyoA from actin, as measured by light scattering, is limited by the rate of ADP release from the motor (Fig. 5d, Supplementary Table 4). For phosphorylated WT, S19A, and K764E, the rate of ADP release was faster than the steady-state ATPase rate. Interestingly, deletion of the $\mathrm{N}$-term extension caused ADP release to become the rate-limiting step in the cycle $\left(\sim 10 \mathrm{~s}^{-1}\right)$, a hallmark of high duty-ratio motors that spend the majority of their ATPase cycle in a state strongly bound to actin ${ }^{30}$.

Ensemble force measurements using a utrophin-based loaded motility assay and automated filament-tracking software ${ }^{31}$ showed that $\Delta \mathrm{N}$ produced $\sim 8$-fold more force, and S19A and K764E produced $\sim 2$-fold more force than phosphorylated PfMyoA (Fig. 5e, Supplementary Fig. 7b). These results demonstrate that Ser19 phosphorylation, which has been shown to occur in vivo ${ }^{32}$, likely tunes PfMyoA motor properties. The absence of Ser19 phosphorylation, or removal of the N-term extension, enhances ensemble force at the expense of speed, confirming the role of this atypical extension in force generation.

The electrostatic bond between SEP19 and ConverterK764 stabilizes the Rigor-like conformation while it is unlikely to form in high ADP affinity states. The rate of the last transition of the powerstroke (ADP release) is thus faster when Ser19 is phosphorylated. Faster ADP/ATP exchange and motor detachment from F-actin leads to higher speed by accelerating motor turn-over. The role of SEP19 in this transition was further investigated in silico using the mutant K764E. Molecular dynamic simulations on a $60 \mathrm{~ns}$ time-course further confirm the role of this electrostatic bond for the stability of the converter position, by comparing phosphorylated WT and K764E mutant in the Rigorlike state (Supplementary Fig. 8). In the WT phosphorylated motor domain, the converter is maintained in its Rigor-like position throughout the simulation (Supplementary Fig. 8a, c, d, Supplementary Movie 5). Conversely, in the K764E mutant, the converter position is not maintained and progressively deviates from its initial position together with the Relay, while the SH1helix stays immobile (Supplementary Fig. 8b, c, d, Supplementary Movie 6). The in silico and in vitro results confirm that the electrostatic interaction between SEP19 and K764 stabilizes the Rigor-like state and is important for modulating ensemble force and the speed at which PfMyoA moves actin.

\section{Discussion}

The divergent force production mechanism of PfMyoA, which we now show underpins the breadth of Plasmodium parasite lifecycle progression, is the result of sequence adaptations in which the $\mathrm{N}$-term extension compensates for the absence of the ${ }^{\mathrm{SH} 1-\mathrm{SH}} 2 \mathrm{Gly}$ fulcrum residue, shown as essential for the piston-like movement of the SH1-helix of canonical myosins ${ }^{18}$. By providing three 
structural states of the PfMyoA motor, our data directly show the atypical mechanism of force production. While the sequence adaptation near the ${ }^{\mathrm{SH} 1-\mathrm{SH} 2} \mathrm{Gly} /$ Wedge was correctly predicted from the TgMyoA PPS structure as part of this mechanism ${ }^{20}$, the authors could not envisage that this would lead to a lack of piston-like movement of the SH1-helix as shown here. Other speculations in the study were incorrect because of an inability to visualize the $\mathrm{N}$-term extension in the TgMyoA structure and the absence of a Rigor-like state structure. The details provided by the PfMyoA structures here, highlight how this $\mathrm{N}$-term extension plays a direct and critical role in the force generation mechanism by direct interactions with Switch-2 and the converter.

The PfMyoA Rigor-like structure reveals how an interaction of the phosphorylated serine in the $\mathrm{N}$-term extension with the converter is directly involved in the last step of the lever arm swing associated with ADP release. In so doing, phosphorylation directly controls the speed and force sensitivity of the PfMyoA motor by modulating the time spent strongly attached to F-actin. These insights into the PfMyoA mechanical cycle reveal that a specific phosphorylation event on class XIV myosins tunes their motor activity by a change in their duty ratio. This allows switching between an ability of the motor to generate force under loaded conditions, or to move actin for highest speed under unloaded conditions. The ATPase activity and unloaded in vitro motility speed of expressed PfMyoA were also recently measured by Green et $\mathrm{al}^{33}$, but with values for both that were over 10 -fold lower than reported here. Although part of this discrepancy $(\sim 2-$ fold) can be attributed to different assay temperatures (our study $30{ }^{\circ} \mathrm{C}$, their study $23^{\circ} \mathrm{C}$ ), and part to not knowing the state of phosphorylation of their expressed myosin (dephosphorylation would slow values 2 -fold), it is unclear why their values are considerably lower than reported here.

In mammalian Myolb, ADP release has been shown to involve the N-terminal extension of the motor that docks at the motor domain/lever arm interface in the Rigor state but is free of interaction in the strong ADP state 27,34 . The position of the N-terminal extension and the location of the bonds that favor the Rigor-like state differ greatly between PfMyoA and Myolb (Supplementary Fig. 10), although in both cases, the presence of the N-terminal extension increases ADP release by forming precise bonds stabilizing the Rigor state. No phosphorylation has been reported for the Myolb N-terminal extension which contains two serine residues (Ser8, Ser9), so it is unknown whether the tuning of ADP release found by phosphorylation of PfMyoA might be recapitulated in other members of the myosin superfamily such as Myolb. If phosphorylation on either of these Myolb serines occurs, it would greatly perturb and likely prevent the docking of the $\mathrm{N}$-terminal extension required for stabilizing the Rigor state. Thus, while both $\mathrm{N}$-terminal extensions contribute to stabilizing the Rigor state, the nature of how this is done differs greatly between these myosins. The PfMyoA N-term extension, in contrast to that of Myolb, is integral to the motor domain and directly influences the connectors that drive the lever arm swing in addition to the position of the converter. Depending on its phosphorylation state, the PfMyoA N-term extension stabilizes the Rigor state in a unique and tunable way to control the motor duty ratio.

The mechanism presented here provides new insights into how PfMyoA may modulate its motor properties and adapt for optimized Plasmodium parasite function across the lifecycle. Liverstage sporozoites can move at speeds of $>2 \mu \mathrm{m} . \mathrm{s}^{-1}$ across substrates or within extra-cellular space. This high speed is one of the fastest characterized for any eukaryotic cell. In contrast, blood stages merozoites, which are relatively immotile away from host cells, require the development of force $(\sim 40 \mathrm{pN})$ by the glideosome against the erythrocytes specifically for invasion ${ }^{4}$. Because it is now clear that PfMyoA is required for both motility and invasion processes, Ser19 phosphorylation could provide a mechanism to optimize motor properties (speed vs. force) depending on parasite life-cycle stage (Fig. 6). From proteomic studies there is evidence of S19 phosphorylation in schizonts, merozoites $^{32}$ and salivary gland sporozoites ${ }^{35}$ but further study with invasion-ready parasites will be required to show the extent of S19 phosphorylation in each stage. Motility studies with liverstage sporozoites would be required to definitively test this hypothesis and how it relates to the subtleties of motor tuning.

All the Apicomplexan parasites differ in their life-cycle, as well as in the host organisms that they target. The function of myosin A amongst these organisms may differ too, as suggested by the differences found for the role and essentiality of MyoA in the genus Plasmodium vs. Toxoplasma. In T. gondii, TgMyoA is essential for egress ${ }^{6,7}$ but not for host cell invasion ${ }^{8,9}$. Here, we demonstrate that PfMyoA is critical for erythrocytic invasion in $P$. falciparum, but the parasite is still able to egress in the absence of GAP45, the protein anchoring PfMyoA to the inner membrane complex ${ }^{13}$. This example shows likely key differences in the cellular basis of host cell entry and exit across Apicomplexan parasites, highlighting that caution should be used when extrapolating conclusions from one genus to another.

These results also raise the question about the conservation of the atypical and tunable mechanism of PfMyoA in other apicomplexan parasites. All the sequence adaptations described here are present in the recently solved PPS structural state of TgMyoA ${ }^{20}$ : the G-to-S substitution at the SH2-SH1 fulcrum; the sequence adaptation to maintain the interaction between the Relay and the SH1-helix; the substitution of the aromatic residue of the Wedge by a threonine. In the TgMyoA converter there is a lysine (K766) equivalent to K764 in PfMyoA. Moreover, the TgMyoA N-term extension contains three serines that can be phosphorylated: S20, S21 that is equivalent to S19 in PfMyoA, and S29. An additional serine can be phosphorylated (S743) in the converter. Phosphomimetic mutants showed that the number of charged residues in the N-terminus modulates the speed at which TgMyoA moved actin filaments ${ }^{20}$, although we showed here that a phosphomimic does not recapitulate the full effect of a phosphorylated Ser. While the N-term extension was not defined in the electron density of the TgMyoA X-ray structure, the sequence conservation is sufficient to assume that it may form a helix and a turn, as found in our PfMyoA structures (Supplementary Fig. 3d, e). The basis of the tunable mechanism described here for PfMyoA is thus likely conserved in TgMyoA, although possibly more complex because it involves two additional phosphorylation sites in the $\mathrm{N}$-term extension and one additional site in the converter. These differences in the possible tuning of PfMyoA and TgMyoA activity may result from variations of the requirement for the glideosome motor during the different life-cycles of the two parasites. Indeed, across its human host, Plasmodium falciparum alternates between the highly motile sporozoite and the invasive but relatively immobile merozoite. In contrast, the free stage of Toxoplasma gondii is the tachyzoite with a speed of $5 \mu \mathrm{m} . \mathrm{s}^{-1}$, similar to the maximum in vitro motility speed for TgMyoA when this myosin had three $\mathrm{N}$-terminal phospho-mimic Asp residues ${ }^{20}$. Tachyzoites, however, can either undergo dissemination, invasion or egress. Thus, controlling the phosphorylation status of TgMyoA in tachyzoites could in principle modulate the speed and/or force developed by TgMyoA and thus adapt its motor properties depending on the need for either the dissemination, invasion or egress phases of the parasite. It may be possible to test this hypothesis with future studies on TgMyoA, permitting a direct comparison to the PfMyoA tuning mechanism revealed here.

Finally, we show here definitively for the first time that PfMyoA is critical for erythrocytic invasion. Myosin A is thus not only 


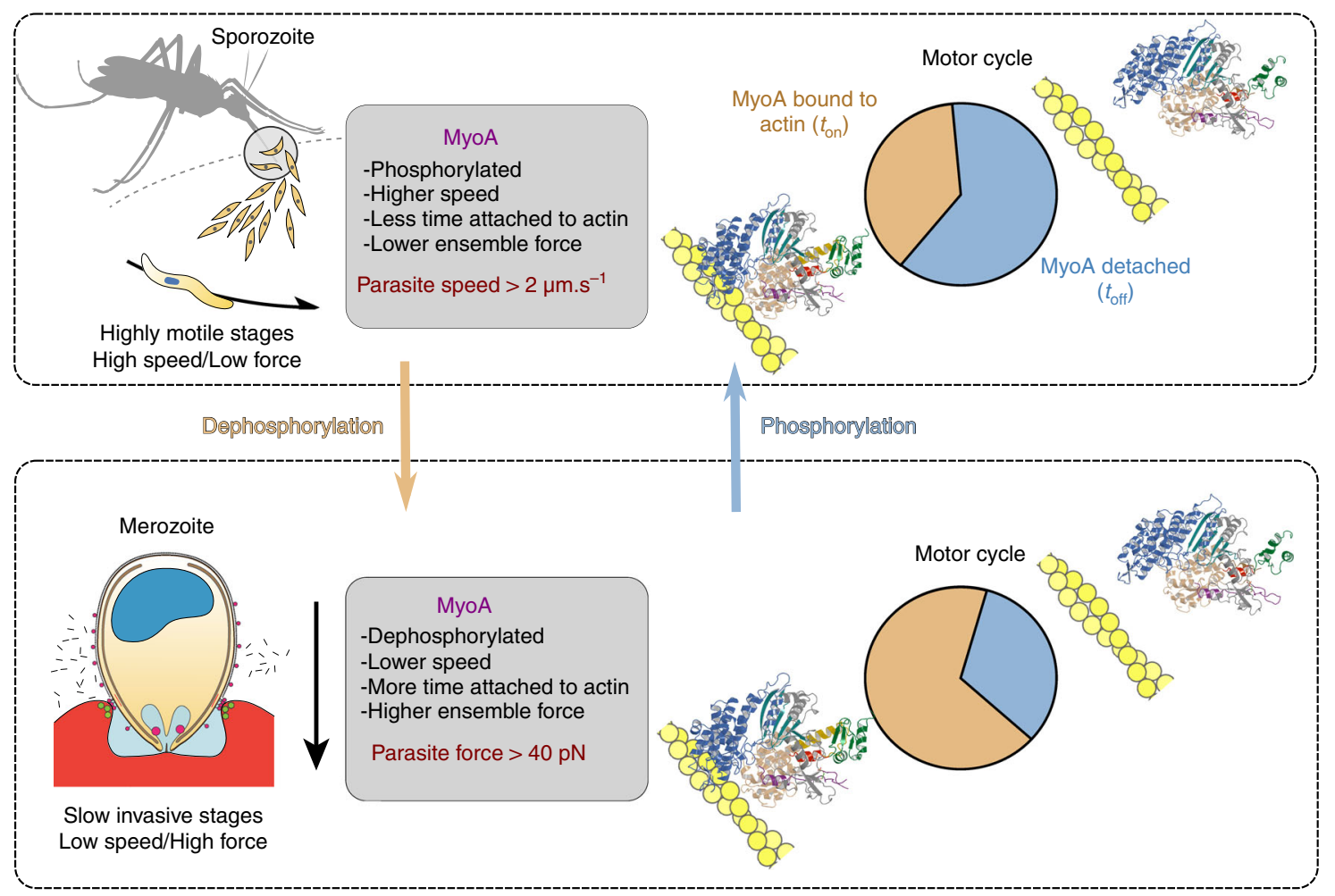

Fig. 6 Phosphorylation of PfMyoA tunes its motor properties. Scheme representing how phosphorylation tunes PfMyoA motor properties and how this could optimize the motor for parasite motility or invasion at different stages of the parasite. In highly motile stages, sporozoites move at a speed higher than $2 \mu \mathrm{m} / \mathrm{s}$. At this stage, phosphorylation of PfMyoA would allow the parasite to move actin at maximal speed but with low ensemble force. In merozoites, which lack continuous motility, but are instead adapted for erythrocyte invasion, dephosphorylation of PfMyoA localized at the invasion junction would result in active motors efficient in invasion. This merozoite PfMyoA motor would spend more of its total cycle time strongly bound to actin, thereby resulting in greater ensemble force output. The apparent duty ratio in the two phosphorylation states was estimated from the rate of ADP release divided by the total ATPase cycle time. The S19A, K764E and $\Delta \mathrm{N}$ mutants would likely impair speed but not invasion, because their ensemble force is higher than phosphorylated, wild-type PfMyoA. Phosphorylation of the N-term extension of PfMyoA can thus act as a switch to tune motor activity depending on the needs of the parasite (high speed and low force for gliding, or higher force for the low speed invasion process)

required for motility in general in the Plasmodium genus but also for establishing blood stage infection during the symptomatic stages. The levels of reinvasion are commensurate with a complete block in invasion, as shown by comparison with CytoD and heparin. Nevertheless, there may be some residual invasion due to parasites with non-excised pfmyoa locus and there is a possibility of invasion by RBC membrane wrapping interactions alone ${ }^{11}$. However, the size of this residual population appears much smaller than found in T. gondii tachyzoites lacking TgMyoA ${ }^{9}$ and would argue against redundancy in motor function like that seen in Toxoplasma.

In recent years, several small molecules targeting myosins have been developed as promising treatments for diverse pathologies such as cardiac diseases and asthma ${ }^{36-39}$. Amongst these modulators, CK-571 is able to selectively block smooth muscle myosin by trapping an intermediate of the recovery stroke ${ }^{38}$. The design of molecules able to block PfMyoA activity based on similar principles, such as that found for CK-571, would be a highly specific and efficient way to treat malaria. A $~ 1000$-fold difference in IC50 allows CK-571 to be tuned for inhibition of smooth muscle myosin compared with cardiac or skeletal muscle myosins, despite the fact that the allosteric drug binding pocket corresponds to conserved residues in sequence among these Myo2 motors. The divergence of PfMyoA in terms of sequence and mechanism would ensure an even higher selectivity for inhibitors of the recovery stroke. The atypical mechanism of PfMyoA described here and the structural blueprints provided should therefore enable the specific design of inhibitors that could stall the progression of malarial infection.

\section{Methods}

Expression constructs. Full-length PfMyoA heavy chain (PlasmoDB ID PF3D7_1342600/ GenBank accession number XM_001350111.1), with Sf9 cell preferred codons, was cloned into the baculovirus transfer vector pFastbac (pFB). A 13 amino acid linker separates the C-terminus of the PfMyoA heavy chain from an 88 amino acid segment of the Escherichia coli biotin carboxyl carrier protein ${ }^{40}$, which gets biotinated during expression in Sf9 cells, followed by a C-terminal FLAG tag for purification via affinity chromatography. The S19A or K764E mutated versions were generated using site directed mutagenesis. The $\Delta \mathrm{N}$ version of full length PfMyoA heavy chain was made by removing residues Ala2 through Ser19. All PCR products were cloned into the baculovirus transfer vector pAcSG2 (BD Biosciences) to make recombinant virus for the Sf9 expression system. The mouse utrophin (NP_035812) clone was a gift from Kathleen Ruppel and James Spudich. It was modified so that utrophin residues 1-H416 were followed by C-terminal biotin and FLAG tags. It was cloned into pFastbac for production of recombinant baculovirus and subsequent expression in Sf9 cells.

Protein expression and purification. The full-length PfMyoA heavy chain or mutant constructs (S19A, K764E, $\Delta \mathrm{N}$ ) were co-expressed with the chaperone PUNC and the light chains (PfMTIP and PfELC) in Sf9 cells as described in ref. ${ }^{19}$. The cells were grown for $72 \mathrm{~h}$ in medium containing $0.2 \mathrm{mg} / \mathrm{ml}$ biotin, harvested and lysed by sonication in $10 \mathrm{mM}$ imidazole, $\mathrm{pH} 7.4,0.2 \mathrm{M} \mathrm{NaCl}, 1 \mathrm{mM}$ EGTA, $5 \mathrm{mM} \mathrm{MgCl} 2,7 \%$ (w/v) sucrose, $2 \mathrm{mM}$ DTT, $0.5 \mathrm{mM}$ 4-(2-aminoethyl)benzenesuflonyl fluoride, $5 \mu \mathrm{g} / \mathrm{ml}$ leupeptin, $2 \mathrm{mM}$ MgATP. An additional $2 \mathrm{mM} \mathrm{MgATP}$ was added prior to a clarifying spin at $200,000 \times g$ for $40 \mathrm{~min}$. The supernatant was purified using FLAG-affinity chromatography (Sigma). The column was washed with $10 \mathrm{mM}$ imidazole $\mathrm{pH} 7.4,0.2 \mathrm{M} \mathrm{NaCl}$, and $1 \mathrm{mM}$ EGTA and the myosin eluted from the column using the same buffer plus $0.1 \mathrm{mg} / \mathrm{ml}$ FLAG peptide. The fractions containing myosin were pooled and concentrated using an Amicon centrifugal filter device (Millipore), and dialyzed overnight against $10 \mathrm{mM}$ imidazole, pH 7.4, $0.2 \mathrm{M} \mathrm{NaCl}, 1 \mathrm{mM}$ EGTA, 55\% (v/v) glycerol, $1 \mathrm{mM} \mathrm{DTT}$, and $1 \mu \mathrm{g} / \mathrm{ml}$ leupeptin and stored at $-20^{\circ} \mathrm{C}$. Utrophin purification was essentially the same as for myosin but without the MgATP steps. Skeletal muscle actin was purified from chicken skeletal muscle tissue essentially as described in ${ }^{41}$. 
His-tagged PfMTIP and PfELC were bacterially expressed using BLR(DE3)competent cells and the isopropyl $\beta$-D-thiogalactopyranoside induction system ${ }^{40}$. Pellets were lysed by sonication in $10 \mathrm{mM}$ sodium phosphate, $\mathrm{pH} 7.4,0.3 \mathrm{M} \mathrm{NaCl}$, $0.5 \%$ (v/v) glycerol, $7 \%(\mathrm{w} / \mathrm{v})$ sucrose, $7 \mathrm{mM} \beta$-mercaptoethanol, $0.5 \mathrm{mM} \mathrm{4-(2-}$ aminoethyl)benzenesuflonyl fluoride, and $5 \mu \mathrm{g} / \mathrm{ml}$ leupeptin. The cell lysate was clarified at $26,000 \times \mathrm{g}$ for $30 \mathrm{~min}$. The supernatant was boiled for $10 \mathrm{~min}$ in a double boiler, clarified at $26,000 \times g$ for $30 \mathrm{~min}$, and the supernatant loaded on a His-select nickel-affinity column (Sigma). The resin was washed in $10 \mathrm{mM}$ sodium phosphate, $\mathrm{pH} 7.4,0.3 \mathrm{M} \mathrm{NaCl}$ before being eluted in the same buffer containing $200 \mathrm{mM}$ imidazole. The protein was concentrated and dialyzed overnight against $10 \mathrm{mM}$ imidazole, $\mathrm{pH}$ 7.4, $150 \mathrm{mM} \mathrm{NaCl}, 1 \mathrm{mM}$ EGTA, $1 \mathrm{mM} \mathrm{MgCl} 2,55 \%(\mathrm{v} / \mathrm{v})$ glycerol, $1 \mathrm{mM}$ DTT and stored at $-20^{\circ} \mathrm{C}$.

Crystallization and data processing. Crystals of PfMyoA motor domain in Postrigor and Rigor-like states (type 1 crystals) $\left(10 \mathrm{mg} \cdot \mathrm{ml}^{-1}\right)$ were obtained at $4{ }^{\circ} \mathrm{C}$ by the hanging drop vapor diffusion method from a 1:1 mixture of protein with $2 \mathrm{mM}$ EGTA and precipitant containing 18\% PEG 3350; $100 \mathrm{mM}$ glycine $\mathrm{pH}$ 8.6. Crystals of PfMyoA motor domain in Pre-powerstroke state (type 2 crystals) were obtained at $17^{\circ} \mathrm{C}$ by the hanging drop vapor diffusion method from a 1:1 mixture of protein (21 mg.ml ${ }^{-1}$ ) with $2 \mathrm{mM} \mathrm{MgADP} . \mathrm{VO}_{4}$ and precipitant containing $0.6 \mathrm{M} \mathrm{K}$ Phosphate dibasic and $0.7 \mathrm{M}$ Na-Phosphate monobasic. Crystals were transferred in the mother liquor containing 30\% glycerol before flash freezing in liquid nitrogen. X-ray diffraction data were collected at $100 \mathrm{~K}$ on the PX1 $(\lambda=0.906019$ $\AA$ ) for type 1 crystals and on the PX2 $(\lambda=0.979384 \AA)$ for type 2 crystals, at the SOLEIL synchrotron. Diffraction data were processed using the XDS package ${ }^{22}$ and AutoProc ${ }^{43}$. Data obtained from the crystal type 2 were anisotropic, processing has been performed with StarAniso ${ }^{44}$. The crystals type 1 belong to the $\mathrm{P} 2{ }_{1}$ space group with four molecules per asymmetric unit. The type 2 crystals belong to the $\mathrm{P}_{1} 22$ space group with one molecule per asymmetric unit. The cell parameters and data collection statistics for these crystals are reported in Supplementary Tables 1 and 2 , respectively.

Structure determination and refinement. Molecular replacement in type 1 crystals was performed with the motor domain (residues 66-688) of scallop myosin 2 in Post-rigor state (PDB code 1B7T) and in Rigor-like state (PDB code 2OS8) without water and ligand with Phaser ${ }^{45}$ from the CCP4i program suite. Manual model building was achieved using $\operatorname{Coot}^{46}$. Refinement was performed with Bus$\operatorname{ter}^{47}$. The statistics for most favored, allowed and outlier Ramachandran angles are $95.63,3.97$, and $0.4 \%$, respectively. Structure determination indicated that this crystal form corresponds to three molecules in the Post-rigor state (chains A, B, C) and one molecule in the Rigor-like state (chain D). The final model has been deposited on the PDB (PDB code 6I7D). The procedure for structure determination and refinement for crystals type 2 was identical, using one molecule of PfMyoA in Post-rigor state without the converter (residues 2-704) and without water and ligand as search model for molecular replacement. The statistics for most favored, allowed and outlier Ramachandran angles are $82.49,13.40$, and $4.11 \%$, respectively. Structure determination indicated that this crystal form corresponds to the Pre-powerstroke state. The final model has been deposited on the PDB (PDB code 6I7E).

Molecular dynamics simulations. The procedure used for molecular dynamics simulation is similar to what was described in $^{48}$ Inputs were prepared with the CHARMM-GUI ${ }^{49}$ with the Quick MD Simulator module ${ }^{50}$. The CHARMM36 force field ${ }^{51}$ was used to describe the full systems in a box with explicit water (TIP3P model) and salt ( $\mathrm{KCl}$ reaching $150 \mathrm{mM}$ ). The protocol provided in the Quick MD output was followed. Gromacs ${ }^{52}$ (VERSION 5.0-rcl) was used to execute the $60 \mathrm{~ns}$ simulations. Each molecular dynamics simulation was analyzed with the Gromacs tools.

Motor cycle reconstitution and visualization. In this study, we reconstitute the motor cycle based on three structural states: Post-rigor (PR), Pre-powerstroke (PPS) and Rigor-like. To visualize structural changes during the powerstroke, we used a comparison between the PPS and the Rigor-like states. We have made some animations (Supplementary Movies 1-4) to compare the conformation of the different connectors in the PPS and in the Rigor-like in conventional myosins (ScMyo2) and in the atypical PfMyoA. Morphs were not used to illustrate the transition between the PPS and the Rigor-like states since the isomerization occurs through intermediate states ${ }^{53}$ which are not known for these myosins, this kind of procedure would thus be misleading.

In order to visualize the powerstroke of a conventional class II myosin, we have used scallop myosin II (ScMyo2). The structures of the Pre-powerstroke (PPS) and of the Rigor-like states of ScMyo2 have been solved in different organisms: PPS of bay scallop (Argopecten irradians) ScMyo2 (PDB code 1QVI, resolution 2.54 $\AA$ ) and Rigor-like state of Atlantic deep-sea scallop (Placopecten magellanicus) ScMyo2 (PDB code 2OS8, resolution 3.27 ). The sequence of ScMyo2 heavy chain in these two species is highly conserved for the motor domain (92\% identity, $95 \%$ similarity) and the two structures can thus be used to reconstitute the powerstroke of ScMyo2.
Unloaded and loaded in vitro motility measurements. Before the assay, myosin heads that cannot release from actin upon binding MgATP were removed by a 20 min spin at $350,000 \times g$ in the presence of $1.5 \mathrm{mM} \mathrm{MgATP}$ and a three-fold excess of skeletal actin. Myosin and utrophin (when applicable) concentrations were determined using the Bio-Rad Protein assay. The following solutions were added to a nitrocellulose-coated flow cell in $15 \mu \mathrm{l}$ volumes. $0.5 \mathrm{mg} / \mathrm{ml}$ biotinylated bovine serum albumin (BSA) in buffer A ( $150 \mathrm{mM} \mathrm{KCl}, 25 \mathrm{mM}$ imidazole pH 7.5, $1 \mathrm{mM}$ EGTA, $4 \mathrm{mM} \mathrm{MgCl}_{2}$, and $10 \mathrm{mM}$ DTT) was added and incubated for $1 \mathrm{~min}$. Three additions of $0.7 \mathrm{mg} / \mathrm{ml} \mathrm{BSA}$ in buffer A and a 2 min wait prevent subsequent nonspecific binding of either the myosin or utrophin. $25 \mu \mathrm{g} / \mathrm{ml}$ neutravidin (Thermo Fischer Scientific) in buffer A was added for $1 \mathrm{~min}$ and then rinsed three times with buffer A. $120 \mu \mathrm{g} / \mathrm{ml}$ PfMyoA (and utrophin, when performing a loaded in vitro motility assay) was flowed into the cell and rinsed 3 times with buffer A after a 1 min wait. All myosin and utrophin constructs contained a C-terminal biotin tag for specific attachment to the neutravidin coated flow cell. Rhodaminephalloidin labeled skeletal muscle actin was incubated for $30 \mathrm{~s}$ followed by one rinse with buffer A and one rinse with buffer B. Buffer B is buffer A plus $0.5 \%(w / v)$ methylcellulose, $25 \mu \mathrm{g} / \mathrm{ml}$ PfELC and $25 \mu \mathrm{g} / \mathrm{ml}$ PfMTIP and oxygen scavengers ( 50 $\mu \mathrm{g} / \mathrm{ml}$ catalase (Sigma), $125 \mu \mathrm{g} / \mathrm{ml}$ glucose oxidase (Sigma), and $3 \mathrm{mg} / \mathrm{ml}$ glucose) Motility was initiated by adding buffer B containing $2 \mathrm{mM}$ MgATP twice to the flow cell, and waiting $1 \mathrm{~min}$ for temperature equilibration $\left(30^{\circ} \mathrm{C}\right)$ on the microscope.

Actin filaments were visualized using an inverted microscope (Zeiss Axiovert 10) equipped with epifluorescence, a Rolera MGi Plus digital camera, and a dedicated computer with the Nikon NIS Elements software package. Filaments were automatically tracked and analyzed, both under unloaded and loaded conditions, with the Fast Automated Spud Trekker analysis program (FAST, Spudich laboratory, Stanford University). This online software is available free for download (http://spudlab.stanford.edu/fast-for-automatic-motility-measurements/).

Speeds for all unloaded motility experiments were fit to a Gaussian curve. All loaded motility data sets were fitted to the equation:

$$
\text { Time mobile }=\frac{K_{S} M_{0}}{K_{S}+M_{0}[\text { utrophin }]}
$$

Where $M_{\mathrm{o}}$ is percent mobile filaments in unloaded conditions, $K_{\mathrm{S}}$ is a pseudo actinutrophin dissociation constant (in $\mathrm{nM}$ ), that is sensitive to myosin ensemble force, and $\%$ time mobile is the total duration of filaments in the mobile state divided by the total duration of the combined stuck and mobile states (see ref. ${ }^{31}$ for a detailed explanation).

Actin-activated ATPase activity. The actin-activated ATPase activity of all PfMyoA constructs was determined at $30^{\circ} \mathrm{C}$ in $10 \mathrm{mM}$ imidazole $\mathrm{pH} 7.5,5 \mathrm{mM}$ $\mathrm{KCl}, 1 \mathrm{mM} \mathrm{MgCl} 2,1 \mathrm{mM}$ EGTA, $1 \mathrm{mM}$ DTT, and $1 \mathrm{mM} \mathrm{NaN}_{3}$ as a function of skeletal actin concentration. The salt concentration was kept low $(5 \mathrm{mM})$ to minimize $K_{\mathrm{m}}$ values and reach $V_{\max }$ with actin concentrations that are compatible with this assay. Actin-activated ATPase activity was determined using a linked assay, which couples the regeneration of hydrolyzed ATP to the oxidation of $\mathrm{NADH}$ to NAD+. Pyruvate kinase (Sigma), in the presence of phophoenolpyruvate (Sigma) and low ADP concentrations, regenerates ATP. In a subsequent reaction, pyruvate is converted to lactate by L-lactate dehydrogenase (Sigma) which oxidizes NADH (Sigma) to NAD +. The decrease in optical density at $340 \mathrm{~nm}$ as a function of time was measured on a Lambda 25 UV/VIS spectrophotometer (Perkin Elmer), with a $300 \mathrm{~s}$ data monitoring window sampled every $2 \mathrm{~s}$. Data were fit to the Michaelis-Menten equation.

Transient kinetics. All kinetic experiments were performed in $10 \mathrm{mM} \mathrm{4-(2-}$ hydroxyethyl)-1-piperazineethanesulfonic acid, $\mathrm{pH} 7.5,50 \mathrm{mM} \mathrm{KCl}, 4 \mathrm{mM} \mathrm{MgCl}_{2}$, $1 \mathrm{mM}$ EGTA, and $1 \mathrm{mM}$ DTT at $30^{\circ} \mathrm{C}$ using a KinTek stopped-flow apparatus (KinTek Corporation, model SF-2001). All concentrations stated are those after mixing in the stopped-flow cell. Light scattering at $295 \mathrm{~nm}$ was used to monitor the dissociation of the actomyosin.ADP complex (skeletal actin) by $2 \mathrm{mM} \mathrm{MgATP}$. Light scattering of unlabeled acto-PfMyoA was observed with an incident beam of $295 \mathrm{~nm}$ and detected orthogonal to the incident beam with a $295 \mathrm{~nm}$ interference filter. All traces were analyzed using the software provided by KinTek and fit to single exponential fits. Typically, multiple (3-10) time courses were averaged before fitting to an exponential.

Mass spectrometry. The phosphorylation status of expressed PfMyoA was determined by liquid chromatography tandem mass spectrometry (LCMS/MS). Aliquots of PfMyoA heavy chain $(104 \mathrm{kDa})$ and PfMyoA heavy chain treated with dephosphorylation with calf intestinal phosphatase (CIP; New England Biolabs, M0290S) were separated by SDS-PAGE, stained with Coomassie and excised from the gel. The bands were destained with 50\% acetonitrile (ACN), dehydrated with $100 \%$ ACN and dried in a speed vacuum device. The bands were destained, reduced, alkylated and digested with $2 \mu \mathrm{g}$ of trypsin (Promega) as decribed ${ }^{19}$. The resultant peptides were dried in a speed vacuum device and then reconstituted in $0.05 \%$ trifluoroacetic acid. The peptides were separated on an Acquity UPLC HSS T3 column (100 ̊, $1.8 \mu \mathrm{m}, 1 \mathrm{~mm} \times 150 \mathrm{~mm})$ (Waters) attached to a Dionex UltiMate 3000 high pressure liquid chromatography system (HPLC) (Dionex). The 
HPLC effluent was directly injected into a Q Exactive Hybrid Quadrupole-Orbitrap mass spectrometer through an electrospray ionization source (ThermoFisher). Data were collected in data-dependent MS/MS mode with the top 5 most abundant ions being selected for fragmentation.

Peptides were identified from the resultant MS/MS spectra using SEQUEST run via Proteome Discoverer 2.2 software (ThermoFisher). These searches were performed against a custom database that reflected the cloned gene (PfMyoA heavy chain) plus tags. Peptide oxidation was accounted for by addition of 15.99 and 31.99 $\mathrm{Da}$ to each methionine, carbamidomethylation was accounted for by addition of 57.02 Da to each cysteine, and phosphorylation was accounted for by adding $79.97 \mathrm{Da}$ to each serine, threonine or tyrosine residue. All identifications were manually confirmed by inspection of the MS/MS fragmentation spectra. LC peak areas were quantified for each peptide using Proteome Discoverer 2.2. The LC peak areas were normalized using the median abundance of the top 5 most abundant PfMyoA peptides. The degree of phosphorylation was estimated from abundance of non-phosphorylated VSNVEAFDK peptide (containing serine 19) in the untreated PfMyoA samples relative to that in the samples dephosphorylated with CIP using a mass-balance approach ${ }^{54}$.

DNA manipulation. For generation of Myo-LP constructs, synthetic gene fragments were ordered (GENEWIZ or Thermo Scientific) comprising the re-codon optimized myosin sequence, loxPint module, tags and T2A skip peptide with $30 \mathrm{bp}$ Gibson flanks. These were assembled with a $\sim 700$ bp long homology region (amplified from 3D7 genomic DNA) by Gibson assembly. The parent vector (pARL-FIKK10.1) was a gift of Dr Moritz Treeck (Crick Institute). Plasmids were purified from E. coli cultures using QIAGEN Plasmid Maxi kit.

P. falciparum culture and transfection. Strain $\mathrm{B} 11^{13}$, which constitutively expresses inducible DiCre, was cultured in RPMI 1640 (Life Technologies) under standard condition $^{55}$ at $4 \%$ hematocrit and synchronized with $5 \%$ sorbitol (Sigma) ${ }^{56}$. Parasites were grown to $5 \%$ at ring stage and electroporated with $100 \mu \mathrm{g}$ of plasmid in $15 \mu \mathrm{L}$ of sterile TE buffer added to $385 \mu \mathrm{L}$ sterile Cytomix ${ }^{57}$. Plasmid uptake was selected for by adding fresh media with $3 \mathrm{nM}$ WR99210 daily for 7 days, then every $2-3$ days until parasite population re-established. Transfectants were grown to $2-4 \%$ parasitaemia and integration was selected for with $400 \mu \mathrm{g} / \mathrm{mL} \mathrm{G} 418^{21}$. Fresh media with G418 was added for 10 days before parasites were returned to drug-free media. MyoA-cKO parasites were cloned by limiting dilution.

\section{Genotyping, western blotting, and immunofluorescence assays. DNA was} extracted using the PureLink Genomic DNA Mini kit (Invitrogen) for genotyping. Schizonts at $\sim 5 \%$ parasitaemia were lysed using $0.1 \%$ saponin/PBS (Sigma) and prepared for Western Blotting by standard methods. The presence of FLAG (F1804, Sigma) or GFP (Roche) was probed using primary antibody at 1:1000 dilution with the appropriate HRP-coupled secondary antibody added at 1:3000 dilution. Detection was performed with ECL (Amersham).

Schizonts at $5 \%$ parasitaemia or higher were treated with cysteine protease inhibitor E64 $(10 \mu \mathrm{M})$ for $4-6 \mathrm{~h}$ to prevent egress, then fixed with 4\% PFA/0.025\% glutaraldehyde/PBS for $1 \mathrm{~h}$, permeabilised with $0.1 \%$ Triton X-100/PBS for $10 \mathrm{~min}$ and blocked overnight at $4{ }^{\circ} \mathrm{C}$ in $3 \%$ BSA/PBS. Fixed cells were incubated with $\alpha$ FLAG (F1804, Sigma), $\alpha$ GAP45 $5^{58}$ and aGFP antibodies (Roche) at 1:500 dilution in $3 \% \mathrm{BSA} / \mathrm{PBS}$ for $2 \mathrm{~h}$, then washed three times in PBS. Secondary antibodies were added at 1:1000 dilution in 3\% BSA/PBS and incubated for $1 \mathrm{~h}$, then cells were washed three times in PBS. The cells were resuspended to a hematocrit of $10 \%$ and mounted with DAPI-VECTASHIELD (Vectorlabs). Images were acquired with an OrcaFlash4.0 CMOS camera using a Nikon Ti Microscope (Nikon Plan Apo $100 \times$ 1.4-N.A. oil immersion objective). Z-stacks were acquired with a step size of $0.2 \mu \mathrm{m}$ and images were deconvoluted using the EpiDEMIC plugin with 50 iterations in Icy $^{59}$. Subsequent image manipulations were carried out in ImageJ2 $2^{60-62}$.

\section{Conditional myosin knockout and quantification. $30 \mathrm{ml}$ of synchronous, late} stage parasites at $5 \%$ parasitaemia were purified by density gradient centrifugation with $70 \%$ Percoll supplemented with sorbitol ${ }^{63}$. The purified schizonts were incubated with fresh RBCs at $1 \%$ hematocrit for $2-3 \mathrm{~h}$, shaking at $100 \mathrm{rpm}$. Once new rings reached $>10 \%$ parasitaemia the remaining schizonts were lysed by sorbitol treatment and rings were split equally into two $10 \mathrm{ml}$ dishes at $2-4 \%$ parasitaemia, $4 \%$ hematocrit and incubated for $16 \mathrm{~h}$ with $0.05 \%$ DMSO or rapamycin $(100 \mathrm{nM}$, Sigma), as well as heparin to prevent any further invasion (Pfizer, 1:25 dilution). Cultures were washed twice in complete media to remove heparin and DMSO/RAP, then transferred to 96 well plates in triplicate at $2 \%$ parasitaemia, $0.15 \%$ hematocrit for quantification, with invasion inhibitors heparin (1:25) or CytoD $(500 \mathrm{nM})$ added to control samples. Parasites were allowed to proceed to the following cycle, and at $16-24 \mathrm{~h}$ post invasion were stained with SYBR Green I (Sigma) at 1:5000 dilution for $15 \mathrm{~min}$. After three washes in PBS the parasites were analyzed by flow cytometry. Gates were set for RBCs, single cells and SYBR Greenpositive cells (Supplementary Fig. 11).

Reporting summary. Further information on research design is available in the Nature Research Reporting Summary linked to this article.

\section{Data availability}

All data are available from the corresponding author upon reasonable request. The crystallographic structures can be found on the Protein Data Bank (PDB codes 6I7D and 6I7E). The source data underlying Figs. 1e, 5b-e and Supplementary Fig 7 are provided as a Source Data file.

Received: 22 November 2018 Accepted: 11 June 2019 Published online: 23 July 2019

\section{References}

1. WHO. World Malaria Report 2017. World Health Organization https://doi. org/10.1016/S0264-410X(07)01183-8 (2017).

2. Frenal, K., Dubremetz, J.-F., Lebrun, M. \& Soldati-Favre, D. Gliding motility powers invasion and egress in Apicomplexa. Nat. Rev. Microbiol. 15, 645-660 (2017).

3. Imwong, M. et al. The spread of artemisinin-resistant Plasmodium falciparum in the Greater Mekong subregion: a molecular epidemiology observational study. Lancet Infect. Dis. 17, 491-497 (2017).

4. Crick, A. J. et al. Quantitation of malaria parasite-erythrocyte cell-cell interactions using optical tweezers. Biophys. J. 107, 846-853 (2014).

5. Münter, S. et al. Plasmodium sporozoite motility is modulated by the turnover of discrete adhesion sites. Cell Host Microbe 6, 551-562 (2009).

6. Meissner, M., Schluter, D. \& Soldati, D. Role of Toxoplasma gondii myosin A in powering parasite gliding and host cell invasion. Science 298, 837-840 (2002).

7. Andenmatten, N. et al. Conditional genome engineering in Toxoplasma gondi uncovers alternative invasion mechanisms. Nat. Methods 10, 125-127 (2013).

8. Frenal, K., Marq, J.-B., Jacot, D., Polonais, V. \& Soldati-Favre, D. Plasticity between MyoC- and MyoA-glideosomes: an example of functional compensation in Toxoplasma gondii invasion. PLoS Pathog. 10, e1004504 (2014).

9. Egarter, S. et al. The toxoplasma Acto-MyoA motor complex is important but not essential for gliding motility and host cell invasion. PLOS ONE 9, e91819 (2014).

10. Bichet, M. et al. Genetic impairment of parasite myosin motors uncovers the contribution of host cell membrane dynamics to Toxoplasma invasion forces. BMC Biol. 14, 97 (2016).

11. Dasgupta, S. et al. Membrane-wrapping contributions to malaria parasite invasion of the human erythrocyte. Biophys. J. 107, 43-54 (2014).

12. Siden-Kiamos, I. et al. Stage-specific depletion of myosin A supports an essential role in motility of malarial ookinetes. Cell. Microbiol 13, 1996-2006 (2011).

13. Perrin, A. J. et al. The actinomyosin motor drives malaria parasite red blood cell invasion but not egress. MBio 9, e00905-18 (2018).

14. Vahokoski, J. et al. Structural differences explain diverse functions of Plasmodium actins. PLoS Pathog. 10, e1004091 (2014).

15. Pospich, S. \& Raunser, S. Single particle cryo-EM-an optimal tool to study cytoskeletal proteins. Curr. Opin. Struct. Biol. 52, 16-24 (2018).

16. Kinose, F., Wang, S. X., Kidambi, U. S., Moncman, C. L. \& Winkelmann, D. A Glycine 699 is pivotal for the motor activity of skeletal muscle myosin. J. Cell Biol. 134, 895-909 (1996).

17. Kad, N. M., Patlak, J. B., Fagnant, P. M., Trybus, K. M. \& Warshaw, D. M. Mutation of a conserved glycine in the SH1-SH2 helix affects the loaddependent kinetics of myosin. Biophys. J. 92, 1623-1631 (2007).

18. Preller, M. et al. Structural basis for the allosteric interference of myosin function by reactive thiol region mutations G680A and G680V. J. Biol. Chem. 286, 35051-35060 (2011).

19. Bookwalter, C. S. et al. Reconstitution of the core of the malaria parasite glideosome with recombinant Plasmodium class XIV myosin A and Plasmodium actin. J. Biol. Chem. 292, 19290-19303 (2017).

20. Powell, C. J. et al. Structural and mechanistic insights into the function of the unconventional class XIV myosin MyoA from Toxoplasma gondii. Proc. Natl Acad. Sci. USA 115, E10548-E10555 (2018).

21. Birnbaum, J. et al. A genetic system to study Plasmodium falciparum protein function. Nat. Methods 14, 450-456 (2017).

22. Jones, M. L. et al. A versatile strategy for rapid conditional genome engineering using loxP sites in a small synthetic intron in Plasmodium falciparum. Sci. Rep. 6, 21800 (2016).

23. Zuccala, E. S. et al. Quantitative phospho-proteomics reveals the Plasmodium merozoite triggers pre-invasion host kinase modification of the red cell cytoskeleton. Sci. Rep. 6, 19766 (2016).

24. Coureux, P.-D. et al. A structural state of the myosin V motor without bound nucleotide. Nature 425, 419-423 (2003).

25. von der Ecken, J., Heissler, S. M., Pathan-Chhatbar, S., Manstein, D. J. \& Raunser, S. Cryo-EM structure of a human cytoplasmic actomyosin complex at near-atomic resolution. Nature 534, 724-728 (2016). 
26. Coureux, P.-D., Sweeney, H. L. \& Houdusse, A. Three myosin V structures delineate essential features of chemo-mechanical transduction. EMBO J. 23, 4527-4537 (2004).

27. Mentes, A. et al. High-resolution cryo-EM structures of actin-bound myosin states reveal the mechanism of myosin force sensing. Proc. Natl Acad. Sci. 115, 1292-1297 (2018).

28. Sweeney, H. L. \& Houdusse, A. Structural and functional insights into the Myosin motor mechanism. Annu. Rev. Biophys. 39, 539-557 (2010).

29. Koppole, S., Smith, J. C. \& Fischer, S. The structural coupling between ATPase activation and recovery stroke in the myosin II motor. Structure 15, 825-837 (2007).

30. De La Cruz, E. M. \& Ostap, E. M. Relating biochemistry and function in the myosin superfamily. Curr. Opin. Cell Biol. 16, 61-67 (2004).

31. Aksel, T., Choe Yu, E., Sutton, S., Ruppel, K. M. \& Spudich, J. A. Ensemble force changes that result from human cardiac myosin mutations and a smallmolecule effector. Cell Rep. 11, 910-920 (2015).

32. Lasonder, E., Green, J. L., Grainger, M., Langsley, G. \& Holder, A. A. Extensive differential protein phosphorylation as intraerythrocytic Plasmodium falciparum schizonts develop into extracellular invasive merozoites. Proteomics 15, 2716-2729 (2015).

33. Green, J. L. et al. Compositional and expression analyses of the glideosome during the Plasmodium life cycle reveal an additional myosin light chain required for maximum motility. J. Biol. Chem. https://doi.org/10.1074/jbc. M117.802769 (2017).

34. Greenberg, M. J., Lin, T., Shuman, H. \& Ostap, E. M. Mechanochemical tuning of myosin-I by the N-terminal region. Proc. Natl Acad. Sci. USA 112, E3337-E3344 (2015)

35. Swearingen, K. E. et al. Proteogenomic analysis of the total and surfaceexposed proteomes of Plasmodium vivax salivary gland sporozoites. PLoS Negl. Trop. Dis. 11, e0005791 (2017).

36. Malik, F. I. et al. Cardiac myosin activation: a potential therapeutic approach for systolic heart failure. Science 331, 1439-1443 (2011).

37. Green, E. M. et al. A small-molecule inhibitor of sarcomere contractility suppresses hypertrophic cardiomyopathy in mice. Science 351, 617-621 (2016).

38. Sirigu, S. et al. Highly selective inhibition of myosin motors provides the basis of potential therapeutic application. Proc. Natl Acad. Sci. USA 113, E7448-E7455 (2016)

39. Planelles-Herrero, V. J., Hartman, J. J., Robert-Paganin, J., Malik, F. I. \& Houdusse, A. Mechanistic and structural basis for activation of cardiac myosin force production by omecamtiv mecarbil. Nat. Commun. 8, 190 (2017).

40. Cronan, J. E. J. Biotination of proteins in vivo. A post-translational modification to label, purify, and study proteins. J. Biol. Chem. 265 , 10327-10333 (1990)

41. Pardee, J. D. \& Spudich, J. A. Purification of muscle actin. Methods Enzymol. https://doi.org/10.1016/0076-6879(82)85020-9 (1982).

42. Kabsch, W. XDS. Acta Crystallogr. Sect. D. Biol. Crystallogr. 66, 125-132 (2010).

43. Vonrhein, C. et al. Data processing and analysis with the autoPROC toolbox. Acta Crystallogr. D. Biol. Crystallogr. 67, 293-302 (2011).

44. Tickle, I. et al. STARANISO. Global Phasing Ltd., Cambridge, United Kingdom (2016). https://doi.org/10.1002/anie.201305315

45. McCoy, A. J. et al. Phaser crystallographic software. J. Appl. Crystallogr. 40, 658-674 (2007).

46. Emsley, P. \& Cowtan, K. Coot: model-building tools for molecular graphics. Acta Crystallogr. Sect. D. Biol. Crystallogr. 60, 2126-2132 (2004).

47. Bricogne, G. et al. BUSTER version 2.10.2. Cambridge, United Kingdom Glob. Phasing Ltd. (2017).

48. Robert-Paganin, J., Auguin, D. \& Houdusse, A. Hypertrophic cardiomyopathy disease results from disparate impairments of cardiac myosin function and auto-inhibition. Nat. Commun. 9, 4019 (2018).

49. Jo, S., Kim, T., Iyer, V. G. \& Im, W. CHARMM-GUI: a web-based graphical user interface for CHARMM. J. Comput. Chem. 29, 1859-1865 (2008).

50. Jo, S. et al. CHARMM-GUI 10 years for biomolecular modeling and simulation. J. Comput. Chem. 38, 1114-1124 (2017).

51. Huang, J. \& MacKerell, A. D. J. CHARMM36 all-atom additive protein force field: validation based on comparison to NMR data. J. Comput. Chem. 34, 2135-2145 (2013).

52. Abraham, M. J. et al. Gromacs: high performance molecular simulations through multi-level parallelism from laptops to supercomputers. SoftwareX 1-2, 19-25 (2015).

53. Houdusse, A. \& Sweeney, H. L. How myosin generates force on actin filaments. Trends Biochem. Sci. 41, 989-997 (2016).

54. Previs, M. J. et al. Quantification of protein phosphorylation by liquid chromatography-mass spectrometry. Anal. Chem. 80, 5864-5872 (2008).

55. Trager, W. \& Jensen, J. B. Human malaria parasites in continuous culture. Science 193, 673-675 (1976)
56. Lambros, C. \& Vanderberg, J. P. Synchronization of Plasmodium falciparum erythrocytic stages in culture. J. Parasitol. 65, 418-420 (1979).

57. Adjalley, S. H., Lee, M. C. S. \& Fidock, D. A. A method for rapid genetic integration into Plasmodium falciparum utilizing mycobacteriophage Bxb1 integrase. Methods Mol. Biol. 634, 87-100 (2010).

58. Baum, J. et al. A conserved molecular motor drives cell invasion and gliding motility across malaria life cycle stages and other apicomplexan parasites. $J$. Biol. Chem. 281, 5197-5208 (2006).

59. de Chaumont, F. et al. Icy: an open bioimage informatics platform for extended reproducible research. Nat. Methods 9, 690-696 (2012).

60. Schindelin, J., Rueden, C. T., Hiner, M. C. \& Eliceiri, K. W. The ImageJ ecosystem: an open platform for biomedical image analysis. Mol. Reprod. Dev. 82, 518-529 (2015).

61. Schindelin, J. et al. Fiji: an open-source platform for biological-image analysis. Nat. Methods 9, 676-682 (2012).

62. Rueden, C. T. et al. ImageJ2: ImageJ for the next generation of scientific image data. BMC Bioinforma. 18, 529 (2017).

63. Radfar, A. et al. Synchronous culture of Plasmodium falciparum at high parasitemia levels. Nat. Protoc. 4, 1899-1915 (2009).

\section{Acknowledgements}

We thank Margaret Titus for critical reading of the paper. We are grateful to beamline scientists of PX1 and PX2A (SOLEIL synchrotron) for excellent support during data collection. We thank Kathleen Ruppel and James Spudich (Stanford University) for the utrophin DNA. We thank Linda Makhlouf for experimental support. This work was funded by National Institutes of Health grant AI 132378 (A.H. and K.M.T.), and HL 124041 (M.J.P.). Parasite work was funded through an Investigator Award (100993/Z/13/ Z, J.B.) and PhD studentship (109007/Z/15/A, T.B.) from Wellcome and through a program grant from the Human Frontier Science Program (RGY0066/2016, A.H. and J.B.). We thank Dr. Stéphane Réty for his advice and help to process the dataset of PfMyoA PPS

\section{Author contributions}

J.R.P., J.P.R., J.B., K.M.T., and A.H. designed the research. J.R.P., G.J., and D.M. crystallized PfMyoA in the different states. J.R.P. solved the structures and performed refinement. D.A. performed molecular dynamics. J.P.R. and C.S.B. performed in vitro functional assays. E.B.K. expressed and purified protein for crystallization. M.J.P. performed mass spectrometry analysis. T.B. generated conditional PfMyoA knockout. J.R.P., J.P.R., J.B., K.M.T., and A.H. discussed the results and wrote the paper with the help of D.A. and D.M.

\section{Additional information}

Supplementary Information accompanies this paper at https://doi.org/10.1038/s41467019-11120-0.

Competing interests: The authors declare no competing interests.

Reprints and permission information is available online at http://npg.nature.com/ reprintsandpermissions/

Peer review information: Nature Communications thanks Stefan Raunser and the other, anonymous, reviewer(s) for their contribution to the peer review of this work. Peer reviewer reports are available.

Publisher's note: Springer Nature remains neutral with regard to jurisdictional claims in published maps and institutional affiliations.

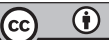

Open Access This article is licensed under a Creative Commons Attribution 4.0 International License, which permits use, sharing, adaptation, distribution and reproduction in any medium or format, as long as you give appropriate credit to the original author(s) and the source, provide a link to the Creative Commons license, and indicate if changes were made. The images or other third party material in this article are included in the article's Creative Commons license, unless indicated otherwise in a credit line to the material. If material is not included in the article's Creative Commons license and your intended use is not permitted by statutory regulation or exceeds the permitted use, you will need to obtain permission directly from the copyright holder. To view a copy of this license, visit http://creativecommons.org/ licenses/by/4.0/

(C) The Author(s) 2019 